\title{
14. A PALEOMAGNETIC STUDY OF SEDIMENTS FROM SITE 397 NORTHWEST AFRICAN CONTINENTAL MARGIN
}

\author{
Norman Hamilton, Department of Geology, University of Southampton, United Kingdom
}

\begin{abstract}
A magnetic polarity stratigraphy is established for the sediments of Site 397, which is located on the northwest African continental margin off Cape Bojador at the continental rise/slope juncture. The magnetic stratigraphy can be correlated with established geomagnetic polarity time-scales by some use of concurrent biostratigraphy control. The combination of magnetic stratigraphy with a good biostratigraphy allows for a more precise dating of important paleoenvironmental events in the development of this part of the northwest African continental margin in Neogene and Quaternary times. Sediment accumulation rates can be inferred from the detailed magnetic stratigraphy.

Below the major hiatus encountered at this site, the Early Cretaceous sediments show predominantly normal magnetic polarity. Correlation to an established polarity time scale indicates probably an uppermost early Hauterivian age.
\end{abstract}

\section{INTRODUCTION}

The continuously cored 1450 -meter sequence of sediments drilled during Leg 47A at Site 397 off Cape Bojador, northwestern Africa (Figure 1) provides a unique opportunity to elucidate a magnetic reversal polarity stratigraphy in a passive continental margin setting. A nearly complete sequence of Quaternary through early Miocene age comprises the principal part of the sediment prism here above a major hiatus, below which occur Early Cretaceous mudstones. It appears that this thick sequence accumulated under conditions of fairly rapid sedimentation throughout much of its history. Such a sequence is ideal for paleomagnetic study as it affords the possibility of preserving a high resolution magnetic stratigraphy. If this magnetic stratigraphy can be accurately deciphered, it will allow a more precise dating of major paleoenvironmental events preserved in the sediment record than can be achieved by using conventional biostratigraphic dating alone.

\section{SAMPLING AND MEASUREMENT}

An essential prerequisite for the determination of a reliable magnetic stratigraphy is a set of sediment samples which give sufficient coverage of the drilled stratigraphic interval so that, commensurate with likely sedimentation rates, no major reversed polarity epochs or events remain undetected. Unfortunately, the practical realization of this prerequisite is constrained by several factors.

1) The condition of the sediments recovered after the coring process.

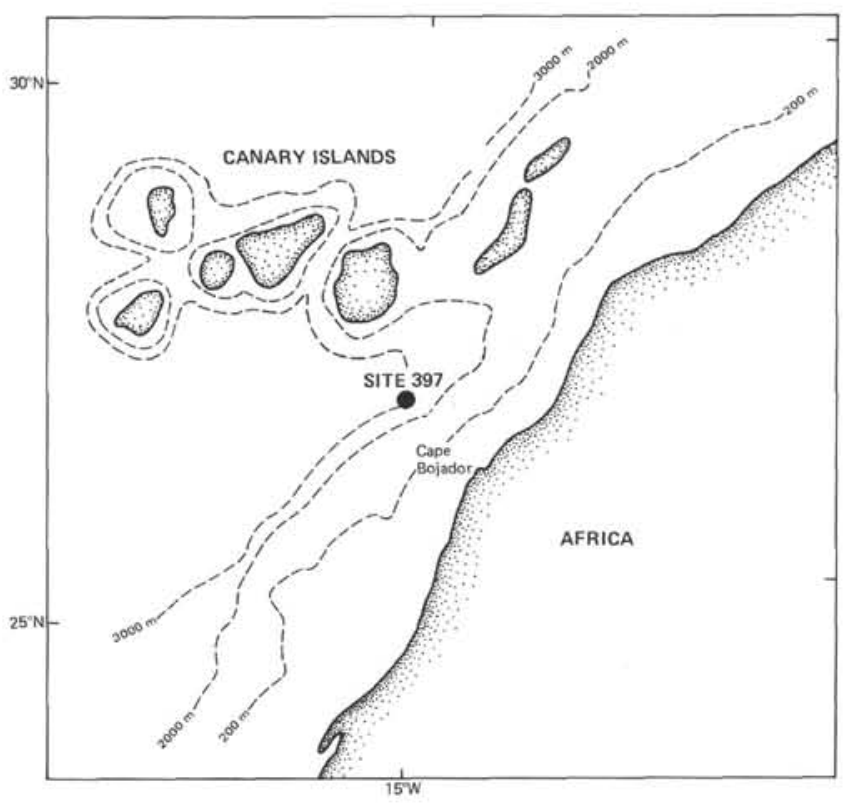

Figure 1. Location of Site 397 on the continental margin off Cape Bojador, Northwest Africa.

Coring deformation is often present in soft unlithified sediments; although it is possible by judicious selection of sampling points to avoid badly disturbed intervals. One major advantage of preliminary shipboard paleomagnetic study, as was undertaken during Leg 47A (see Site 397 Report, this volume), is that sampling and measurement proceed concurrently.

2) The suitability of the drilled lithologies themselves for paleomagnetic study. 
At Site 397, the abundant chaotic pebbly conglomerates of the lower Miocene are not normally suitable for paleomagnetic study. Consequently, sampling is selective in the sub-bottom interval of 900 through 1300 meters. Only the autochthonous pelagic intervals, laminated or graded sand units are chosen for sampling, so there are some gaps in coverage of this part of the sequence. Similarly slumped horizons are avoided. Nevertheless, as these unsampled zones often represent rapidly emplaced allochthonous flows, the apparent time gaps in the stratigraphic intervals may be relatively short.

3) Hiatuses.

The presence and detection of hiatuses in the sedimentary column is also very important if any inferred polarity reversal stratigraphy is to be correlated with established polarity time scales.

4) Remanence type.

The sediments used for paleomagnetic study should possess a natural remanent magnetism (NRM) which was acquired at (or soon after) deposition, thus accurately records the polarity of the ambient geomagnetic field. The isolation of a stable remanence is essential if an unambiguous reversal polarity stratigraphy is to be fully documented.

For this study, 178 samples were obtained by shipboard sampling of the cores recovered from Holes 397 and 397A at Site 397.

Sampling of the Quaternary and late Neogene unlithified marl oozes was achieved by pushing nonmagnetic plastic tubes $(2.5 \mathrm{~cm}$ diameter and $2.2 \mathrm{~cm}$ length which were scribed with orientation marks) vertically into the split core sections. The open ends of the sampling tubes were sealed with adhesive tape after removal from the cores. A standard diamond-tipped core drill was used to extract cylindrical samples from the more lithified sediments. Occasionally, as in the case of of the thinly laminated Early Cretaceous mudstones, cubic-shaped samples were cut from the core segments. The uphole orientation was carefully marked on each sample.

Initial measurements of remanence of the samples were made in the paleomagnetic laboratory established onboard Glomar Challenger at the commencement of Leg 47. A computerized (Digico) spinner magnetometer (Molyneux, 1971) with a noise level of the order of $5 \times 10^{-8} \mathrm{G}$ was used for these measurements. Subsequently, stepwise alternating field demagnetization was accomplished for a number of selected samples of varied lithologies up to peak field values of 400 Oe to investigate stability behavior. Figure 2 gives examples of this procedure for some typical Cenozoic sediment samples. However, for the majority of samples, alternating field demagnetization only up to a peak field of 50 was completed onboard to remove low coercivity components. Shore-based studies continued this progressive demagnetization up to peak fields of 150 and 300 Oe utilizing long signal integration times (commonly $2^{9}$ spins) and triplicate measurement because of a predominance of low intensities of magnetization.
Table 1 gives a summary of the paleomagnetic results for the shipboard sample collection.

For a majority of the samples, low field magnetic susceptibility determinations were made using a Highmoor susceptibility bridge as part of the shorebased study. The results of these measurements are included in Table 1.

\section{RESULTS}

\section{Remanence and Stability Considerations}

\section{Cenozoic Sediments}

NRM measurement of the bulk of the selected samples, which are Quaternary and Neogene in age at Site 397 , reveals a predominance of normal polarity, using inclination as a polarity index. Some 83 per cent of the samples are of normal polarity as shown by the histogram of Figure 3. The histogram of NRM inclinations also has a mode for the distribution which is coincident with the theoretical axial geocentric dipole inclination $\left(45.2^{\circ}\right)$ for this site. As shown in earlier investigations of upper Cenozoic sediments (Amerigian and Watkins, 1975), chemical overprinting of normal polarity in the present geomagnetic field can occur. Evidence of such overprinting is clearly reflected by the distribution of NRM inclination values revealed at this site. Similar findings are described in paleomagnetic studies on sediments from DSDP Sites 379 and 380 in the Black Sea (Hailwood and Hamilton, 1978).

Alternating field demagnetization produces some demonstrable removal of this Brunhes Epoch overprinting, as indicated by other histograms for higher peak field values (Figure 3 ). It seems likely that cleaning at a peak field value of 150 Oe produces a distribution which tends to some bimodality and, therefore, perhaps towards a best estimate of original ambient geomagnetic polarity for these sediments. There is a significant displacement of the modal values to shallower inclinations from that expected for the geocentric axial dipole approximation after demagnetization. Imperfect magnetic cleaning may account to some extent for the shallower mean inclination. Other possible causes for this are known and likely, such as an offset dipole field configuration (Wilson, 1970) or, as Blow and Hamilton (1975) describe, inclination errors can occur to shallow the observed remanence from the ambient field inclination, particularly under conditions of rapid sedimentation with terrigenous input, as might be expected in a continental margin setting. At peak field values $300 \mathrm{oe}$, it is evident that negative inclinations predominate ( $60 \%$ reversed and $40 \%$ normal polarity). This situation is in part an artifact of instrumental effects when demagnetizing at higher peak field values. For many of the samples under investigation, the remanent intensities are then very low (on the order of 5 $\times 10^{8}$ Gauss) so that a reliable direction of magnetization is not easily recognized despite the precaution of triplicate measurement using a longer signal integration time. Nevertheless, the incremental demagnetiza- 

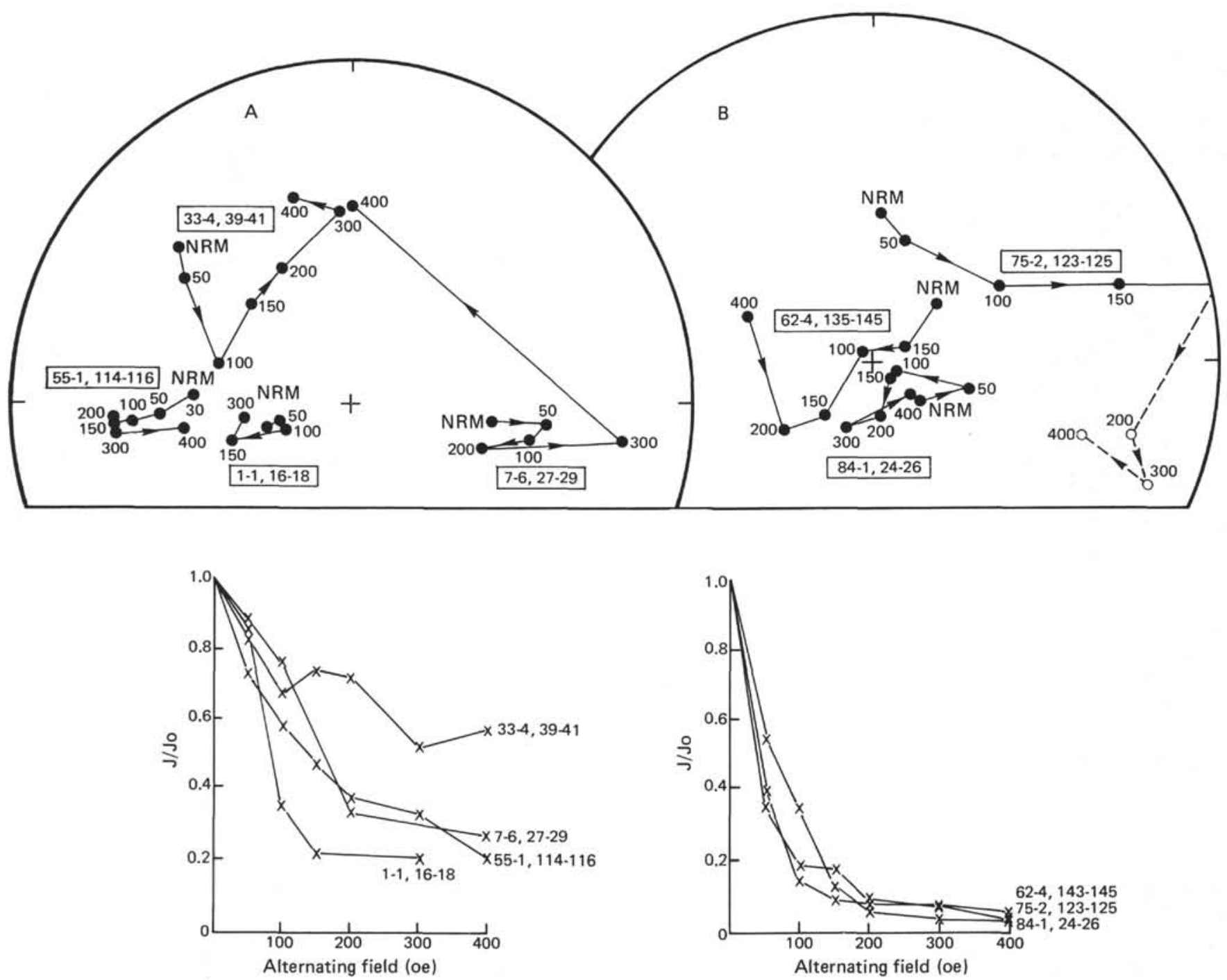

Figure 2. Change in remanence directions during progressive alternating field demagnetization of selected Cenozoic sediment samples from Site 397, together with their associated normalized intensity decay curves: (A) Samples from lithological Units 1 and 2, principally marl oozes or marl chalks, from above a sub-bottom depth of 550 meters. (B) Samples from below 550 meters depth taken from lithological Units 3 and 4. Polar stereographic projection; solid symbols, lower hemisphere (positive inclination); open symbols, upper hemisphere (negative inclination). Numerals in boxes are sample numbers, other numerals are peak alternating field values in Oersted.

tion does seem to have been largely successful in indicating the likely ambient geomagnetic polarity at the time of sediment accumulation. The subsequent interpretation of polarity data for this site is normally based on the inclination given after 150 Oe demagnetization.

It is interesting to note that for those few samples with a reversed polarity NRM, which retain this polarity on demagnetization, a majority occur stratigraphically with the lower Matuyama Epoch. These samples also are characterized by better than average magnetic stability despite their lower remanent intensities. Lithologies of the sediments showing these characteristics are dominantly siliceous marl oozes. No study of the source of the remanence in these lithologies has been undertaken yet.

\section{Mesozoic Sediments}

A sequence of Early Cretaceous mudstones occurs below the major hiatus encountered at 1298 meters sub-bottom at Site 397. Some 155 meters of these sediments were penetrated before drilling terminated. The sediments are thinly bedded and contain alternations of rhythmically laminated dolomicrites. Sedimentological study (Einsele and von Rad, this volume) suggests that the sediments accumulated in a distal prodelta slope environment. The presence of the rhythmic couplets may indicate fairly rapid deposition if an annual periodicity is assumed (see Site 397 Report).

Remanence measurements on 22 samples from this sequence reveal a predominance of positive inclina- 


\section{N. HAMILTON}

TABLE 1 - Continued

\begin{tabular}{|c|c|c|c|c|c|c|c|c|c|c|c|c|c|c|}
\hline $\begin{array}{c}\text { Sample } \\
\text { (Interval in } \mathrm{cm} \text { ) }\end{array}$ & $\begin{array}{l}\text { Depth } \\
\text { (m) }\end{array}$ & $\begin{array}{l}\text { Int. } \\
(\mu G) \\
\pm S . D .\end{array}$ & $\begin{array}{l}\text { NRM } \\
\text { Dec. }\end{array}$ & Inc. & $\begin{array}{l}\text { Int. } \\
(\mu \mathrm{G})\end{array}$ & $\begin{array}{l}50 \text { Oe } \\
\text { Dec. }\end{array}$ & Inc. & $\begin{array}{l}\text { Int. } \\
(\mu \mathrm{G})\end{array}$ & $\begin{array}{c}150 \mathrm{Oe} \\
\text { Dec. }\end{array}$ & Inc. & $\begin{array}{l}\text { Int. } \\
(\mu \mathrm{G})\end{array}$ & $\begin{array}{l}300 \mathrm{Oe} \\
\text { Dec. }\end{array}$ & Inc. & $\begin{array}{l}\text { Susc. } \\
\left(\times 10^{-5}\right. \\
\text { G/Oe })\end{array}$ \\
\hline $68-1,126-128$ & 646.77 & $\begin{array}{r}4.39 \\
\pm 0.14\end{array}$ & 37.9 & +34.0 & 2.05 & 51.1 & +49.9 & 0.48 & 352.2 & +24.4 & 0.37 & 344.0 & +7.2 & 1.91 \\
\hline $68-3,123-125$ & 649.74 & $\begin{array}{l}12.63 \\
\pm 0.31\end{array}$ & 256.9 & +51.7 & 5.62 & 233.0 & +63.6 & 1.61 & 16.4 & +28.2 & 1.20 & 20.4 & +7.7 & 2.15 \\
\hline $69-3,69-71$ & 658.70 & $\begin{array}{l}10.05 \\
\pm 0.26\end{array}$ & 258.3 & +45.3 & 8.71 & 213.2 & +25.0 & 5.68 & 191.9 & +4.7 & 5.13 & 187.8 & -1.0 & 3.66 \\
\hline $70-1,142-144$ & 665.93 & $\begin{array}{r}5.96 \\
\pm 0.11\end{array}$ & 190.9 & +73.8 & 2.99 & 170.8 & +85.0 & 1.26 & 13.9 & +20.0 & 0.39 & 26.7 & +12.2 & 1.85 \\
\hline $70-3,25-29$ & 667.76 & $\begin{array}{r}6.93 \\
\pm 0.18 \\
\end{array}$ & 229.9 & +30.5 & 3.27 & 223.0 & +34.3 & 0.49 & 310.7 & +1.0 & 0.34 & 301.2 & -29.1 & 2.50 \\
\hline $71-1,113-115$ & 675.12 & $\begin{array}{l}23.11 \\
\pm 0.06\end{array}$ & 310.8 & +77.2 & 13.17 & 336.9 & +73.9 & 7.46 & 11.3 & +36.8 & 2.93 & 4.8 & +31.1 & 10.42 \\
\hline $72-1,12-14$ & 683.63 & $\begin{aligned} & 1.03 \\
\pm & 0.07\end{aligned}$ & 274.3 & +10.0 & 0.84 & 268.4 & +5.1 & 0.74 & 284.0 & -1.4 & 0.66 & 270.2 & -11.7 & 2.03 \\
\hline $72-4,4-6$ & 688.05 & $\begin{array}{r}0.37 \\
\pm 0.01\end{array}$ & 39.3 & +30.8 & 0.28 & 43.1 & +22.1 & 0.20 & 6.8 & +23.8 & 0.20 & 17.1 & +15.3 & 0.76 \\
\hline $72-4,46-48$ & 688.49 & $\begin{array}{r}0.20 \\
\pm 0.20\end{array}$ & 62.4 & +53.2 & 0.14 & 215.9 & -38.2 & 0.07 & 125.6 & -27.5 & 0.07 & 162.6 & -33.5 & 0.67 \\
\hline $73-3,16-18$ & 696.17 & $\begin{array}{l}17.99 \\
\pm 0.13 \\
\end{array}$ & 25.3 & +55.3 & 9.47 & 20.9 & +70.0 & 2.07 & 358.3 & +71.1 & 0.45 & 336.1 & +36.6 & 2.80 \\
\hline $73-3,115-117$ & 697.16 & $\begin{array}{r}2.87 \\
\pm 0.31\end{array}$ & 5.3 & +62.0 & 2.33 & 116.5 & +12.6 & 3.64 & 99.5 & -16.8 & 2.66 & 101.4 & -20.1 & 2.01 \\
\hline $74-1,52-54$ & 703.01 & $\begin{array}{r}3.82 \\
\pm 1.81\end{array}$ & 0.7 & +28.6 & 0.85 & 255.8 & +27.7 & 2.39 & 197.9 & -21.1 & 2.11 & 196.3 & -15.7 & 1.75 \\
\hline $75-2,123-124$ & 714.73 & $\begin{array}{r}5.44 \\
\pm 0.01\end{array}$ & 3.8 & +43.5 & 2.18 & 16.3 & +54.1 & 0.46 & 72.5 & +17.6 & 0.45 & 114.0 & -8.3 & 1.66 \\
\hline $76-3,95-97$ & 725.46 & $\begin{array}{r}0.65 \\
\pm 0.01\end{array}$ & 63.0 & +44.4 & 0.85 & 122.0 & +14.0 & 0.86 & 128.2 & -10.6 & - & - & - & 1.11 \\
\hline $77-1,101-105$ & 732.04 & $\begin{array}{r}15.62 \\
\pm 0.39 \\
\end{array}$ & 52.5 & +37.2 & 12.46 & 72.2 & +34.9 & 7.51 & 79.2 & +27.7 & - & - & - & 2.34 \\
\hline $78-1,101-103$ & 741.52 & $\begin{array}{l}10.03 \\
\pm 0.01\end{array}$ & 103.0 & +40.8 & 8.14 & 138.9 & +39.6 & 3.31 & 134.1 & +28.1 & 2.34 & 149.0 & +18.9 & - \\
\hline $78-4, \mathrm{CC}$ & 746.50 & $\begin{array}{r}6.48 \\
\pm 0.09\end{array}$ & 24.9 & +26.7 & 1.70 & 54.5 & +37.8 & 1.27 & 354.7 & -26.2 & 0.48 & 323.1 & -45.4 & 4.51 \\
\hline $79-1,99-101$ & 751.00 & $\begin{array}{r}2.78 \\
\pm 0.01\end{array}$ & 337.8 & +80.0 & 1.82 & 194.1 & +62.5 & 1.29 & 186.7 & +24.1 & 0.73 & 189.6 & +19.2 & 1.55 \\
\hline $79-2,120-122$ & 752.71 & $\begin{array}{l}635.40 \\
\pm 34.00\end{array}$ & 354.9 & +10.1 & 183.92 & 355.3 & +10.0 & 30.98 & 336.2 & -28.5 & 18.47 & 303.0 & -51.1 & - \\
\hline $79-3,6-8$ & 753.07 & $\begin{array}{r}460.70 \\
\pm 30.00 \\
\end{array}$ & 357.7 & +16.3 & - & - & - & 20.56 & 293.2 & +11.1 & 11.65 & 2075 & -23.3 & 94.08 \\
\hline $79-3,105-107$ & 754.06 & $\begin{array}{r}612.00 \\
\pm 25.00\end{array}$ & 351.2 & +11.7 & 120.35 & 324.1 & +4.0 & 56.86 & 241.2 & -27.3 & 32.40 & 230.1 & -50.0 & - \\
\hline $80-1,64-66$ & 760.15 & $\begin{array}{r}210.28 \\
\pm 1.60\end{array}$ & 348.4 & +30.4 & 74.01 & 335.5 & +37.9 & 26.4 & 315.0 & +4.6 & 13.93 & 321.7 & -18.8 & - \\
\hline $80-1,118-120$ & 760.69 & $\begin{array}{r}1.27 \\
\pm 0.01\end{array}$ & 15.7 & +36.0 & 0.39 & 31.6 & +59.3 & 0.09 & 331.9 & +47.0 & 0.77 & 326.9 & -19.1 & 1.29 \\
\hline $80-3,67-69$ & 763.18 & $\begin{array}{c}383.5 \\
\pm 6.3\end{array}$ & 174.7 & +25.9 & - & - & - & 10.78 & 208.6 & +17.9 & 19.80 & 268.7 & -52.9 & 58.06 \\
\hline $83-1,49-51$ & 788.50 & $\begin{array}{r}0.39 \\
\pm 0.02 \\
\end{array}$ & 301.4 & +48.6 & 0.14 & 280.1 & +37.0 & 0.09 & 336.3 & -4.3 & 0.07 & 308.4 & -48.4 & 0.88 \\
\hline $83-1,136-138$ & 789.37 & $\begin{array}{r}0.62 \\
\pm 0.01\end{array}$ & 302.0 & +57.7 & 0.17 & 267.3 & +35.4 & 0.08 & 261.1 & +24.0 & 0.02 & 340.6 & -11.1 & 0.86 \\
\hline $83-2,6-8$ & 789.57 & $\begin{array}{r}0.71 \\
\pm 0.06\end{array}$ & 19.8 & +49.5 & 0.41 & 96.2 & +51.2 & 0.13 & 83.6 & +64.9 & 0.12 & 10.8 & +57.3 & 0.97 \\
\hline $84-1,24-26$ & 797.75 & $\begin{array}{r}105.71 \\
\pm 1.73\end{array}$ & 127.3 & +74.8 & 58.31 & 104.1 & +69.5 & 13.58 & 123.3 & +83.5 & 4.75 & 202.6 & +67.9 & 31.11 \\
\hline $84-2,46-48$ & 799.47 & $\begin{array}{r}25.80 \\
\pm 1.30\end{array}$ & 357.2 & +15.0 & 6.99 & 358.0 & +19.5 & 1.70 & 341.0 & +7.2 & 1.33 & 338.1 & $\begin{array}{r}-4.4 \\
+38.3\end{array}$ & 6.20 \\
\hline $84-2,110-112$ & 800.11 & $\begin{array}{r}127.30 \\
\pm 3.50 \\
\end{array}$ & 340.6 & +47.3 & - & - & - & 32.70 & 289.0 & +48.4 & 19.07 & 287.8 & +38.3 & 36.50 \\
\hline $84-3,113-115$ & 801.62 & $\begin{array}{r}181.00 \\
\pm 6.20\end{array}$ & 353.2 & +18.0 & - & - & - & 32.26 & 358.2 & +19.0 & 18.12 & 358.5 & +8.7 & 34.00 \\
\hline $85-1,68-70$ & 807.69 & $\begin{array}{r}4.53 \\
\pm 0.04\end{array}$ & 176.3 & +83.9 & 0.94 & 356.1 & +85.1 & 0.07 & 320.5 & -25.9 & 0.08 & 259.9 & -45.2 & 4.10 \\
\hline $85-2,132-134$ & 809.83 & $\begin{array}{l}14.30 \\
\pm 1.00\end{array}$ & 348.6 & +21.0 & - & - & - & 1.73 & 290.1 & -25.9 & 1.17 & 250.9 & -37.0 & 5.03 \\
\hline $85-3,8-10$ & 810.09 & $\begin{array}{l}13.50 \\
\pm 0.50\end{array}$ & 1.1 & +38.2 & - & - & - & 1.03 & 344.9 & +9.5 & 1.03 & 354.4 & 44.9 & 5.85 \\
\hline $85-3,114-116$ & 811.15 & $\begin{array}{l}14.90 \\
\pm 0.03\end{array}$ & 2.0 & +21.0 & 1.65 & 18.8 & +55.8 & 1.04 & 179.0 & +35.6 & 0.58 & 207.5 & +1.3 & 5.10 \\
\hline
\end{tabular}


TABLE 1 - Continued

\begin{tabular}{|c|c|c|c|c|c|c|c|c|c|c|c|c|c|c|}
\hline $\begin{array}{c}\text { Sample } \\
\text { (Interval in } \mathrm{cm} \text { ) }\end{array}$ & $\begin{array}{l}\text { Depth } \\
\text { (m) }\end{array}$ & $\begin{array}{l}\text { Int. } \\
(\mu \mathrm{G}) \\
\pm \text { S.D. }\end{array}$ & $\begin{array}{l}\text { NRM } \\
\text { Dec. }\end{array}$ & Inc. & $\begin{array}{l}\text { Int. } \\
(\mu \mathrm{G})\end{array}$ & $\frac{50 \mathrm{Oe}}{\text { Dec. }}$ & Inc. & $\begin{array}{l}\text { Int. } \\
(\mu \mathrm{G})\end{array}$ & $\begin{array}{c}150 \mathrm{Oe} \\
\text { Dec. }\end{array}$ & Inc. & $\begin{array}{l}\text { Int. } \\
(\mu \mathrm{G})\end{array}$ & $\begin{array}{c}300 \mathrm{Oe} \\
\text { Dec. }\end{array}$ & Inc. & $\begin{array}{l}\text { Susc. } \\
\left(\times 10^{-5}\right. \\
\text { G/Oe })\end{array}$ \\
\hline $28-2,22-24$ & 257.73 & $\begin{array}{r}0.18 \\
\pm 0.06\end{array}$ & 335.8 & +7.0 & 0.24 & 47.1 & +74.8 & 0.15 & 31.6 & -48.8 & 0.12 & 57.0 & -56.0 & 0.67 \\
\hline $28-4,84-86$ & 261.35 & $\begin{array}{r}0.41 \\
\pm 0.20\end{array}$ & 59.6 & +2.0 & 0.42 & 356.0 & +47.2 & 0.08 & 357.3 & -25.1 & 0.05 & 348.0 & -64.1 & 0.54 \\
\hline $29-1,13-15$ & 265.64 & $\begin{array}{r}0.13 \\
\pm 0.02\end{array}$ & 105.3 & +4.7 & 0.16 & 72.4 & +21.5 & 0.03 & 151.7 & +66.3 & 0.02 & 185.4 & +44.0 & 0.54 \\
\hline $30-6,86-87$ & 283.37 & $\begin{array}{r}0.15 \\
\pm 0.10\end{array}$ & 324.7 & +8.4 & 0.14 & 34.8 & +31.1 & 0.11 & 338.9 & -5.1 & 0.08 & 347.1 & +10.6 & 0.32 \\
\hline $32-3,61-64$ & 297.62 & $\begin{array}{r}0.16 \\
\pm 0.10 \\
\end{array}$ & 306.6 & +47.3 & 0.17 & 257.9 & -17.5 & 0.51 & 7.9 & -13.9 & 0.06 & 30.0 & -64.6 & 0.41 \\
\hline $33-4,39-41$ & 308.40 & $\begin{array}{r}0.27 \\
\pm 0.08\end{array}$ & 357.3 & +17.4 & 0.16 & 300.0 & +35.0 & 0.19 & 313.4 & +45.8 & 0.13 & 356.0 & +32.0 & 0.38 \\
\hline $34-3,96-98$ & 316.97 & $\begin{array}{r}0.19 \\
\pm 0.07\end{array}$ & 103.4 & +19.6 & 0.12 & 132.4 & +15.1 & 0.21 & 34.9 & +33.1 & 0.03 & 327.8 & +32.0 & 0.38 \\
\hline $36-2,94-96$ & 334.45 & $\begin{array}{r}0.11 \\
\pm 0.03\end{array}$ & 332.6 & +15.7 & 0.12 & 314.3 & +45.0 & 0.07 & 183.0 & -63.0 & 0.06 & 124.1 & -20.2 & 0.26 \\
\hline $37-4,118-120$ & 347.19 & $\begin{array}{r}0.10 \\
\pm 0.04\end{array}$ & 305.6 & +20.4 & 0.11 & 278.5 & +74.4 & 0.06 & 350.2 & +43.4 & 0.03 & 337.4 & -29.6 & 0.31 \\
\hline $38-6,70-72$ & 359.21 & $\begin{array}{r}0.40 \\
\pm 0.06 \\
\end{array}$ & 258.9 & +61.4 & 0.30 & 72.5 & +60.0 & 0.08 & 268.0 & +61.0 & 0.05 & 193.3 & +7.0 & 0.54 \\
\hline $39-4,60-62$ & 365.61 & $\begin{array}{r}0.20 \\
\pm 0.07\end{array}$ & 325.9 & -70.4 & 0.13 & 169.8 & -42.4 & 0.05 & 191.3 & -73.5 & 0.04 & 147.4 & -21.0 & 0.39 \\
\hline $39-5,62-64$ & 367.13 & $\begin{array}{r}0.30 \\
\pm 0.19\end{array}$ & 339.8 & +14.9 & 0.20 & 87.6 & +38.1 & 0.09 & 85.0 & -15.9 & 0.08 & 103.2 & -22.7 & 0.53 \\
\hline $41-7,21-23$ & 388.72 & $\begin{array}{r}0.18 \\
\pm 0.06 \\
0.33\end{array}$ & 231.0 & +15.1 & 0.16 & 302.4 & -8.6 & 0.04 & 52.9 & +11.4 & 0.01 & 318.6 & -78.3 & 0.29 \\
\hline $42-2,39-41$ & 390.90 & $\begin{array}{r}0.33 \\
\pm 0.11 \\
0.18\end{array}$ & 20.1 & -22.4 & 0.33 & 1.3 & +2.9 & 0.04 & 325.2 & -15.9 & 0.03 & 163.7 & $\begin{array}{l}-39.0 \\
+70.6\end{array}$ & 0.48 \\
\hline $43-4,120-122$ & 404.21 & $\begin{array}{r}0.18 \\
\pm 0.09 \\
\end{array}$ & 242.6 & +50.1 & 0.09 & 153.8 & +45.5 & 0.07 & 195.5 & +61.2 & 0.03 & 135.8 & +70.6 & 0.27 \\
\hline $43-5,29-31$ & 404.80 & $\begin{array}{r}0.15 \\
\pm 0.06 \\
0.14\end{array}$ & 251.1 & $\begin{array}{r}-4.4 \\
+64.8\end{array}$ & 0.04 & 310.1 & +58.2 & 0.05 & 3.3 & +205 & 0.01 & 268.5 & $\begin{array}{r}-32.3 \\
+672\end{array}$ & 0.35 \\
\hline $44-2,85-87$ & 410.36 & $\begin{array}{r}0.14 \\
\pm 0.10\end{array}$ & 146.3 & +64.8 & 0.10 & 143.6 & +44.8 & 0.06 & 2.9 & +9.4 & 0.06 & 218.4 & $\begin{array}{l}+67.2 \\
+15.7\end{array}$ & 0.38 \\
\hline $44-2,91-93$ & 410.42 & $\begin{array}{r}0.05 \\
\pm 0.02 \\
0.34\end{array}$ & 181.4 & $\begin{array}{r}+41.8 \\
-13.2\end{array}$ & 0.05 & 156.0 & $\begin{array}{l}+56.4 \\
+271\end{array}$ & 0.08 & 340.8 & $\begin{array}{l}+25.2 \\
-7.6\end{array}$ & 0.04 & 316.5 & +15.1 & 0.46 \\
\hline $47-5,17-18$ & 442.67 & $\begin{array}{r}0.34 \\
\pm 0.08 \\
0.77 \\
\pm 0.05 \\
\end{array}$ & 339.6 & $\begin{array}{r}-13.2 \\
+20.8\end{array}$ & 0.27 & 317.9 & +31.3 & 0.11 & 228.6 & $\begin{array}{r}-1.0 \\
+9.5\end{array}$ & 0.14 & 206.7 & -34.5 & - \\
\hline $47-5,95-97$ & 443.46 & $\begin{array}{r}0.64 \\
\pm 0.02\end{array}$ & 18.2 & -4.2 & 0.21 & 16.3 & -3.6 & 0.17 & 7.2 & -13.3 & 0.05 & 52.7 & $\begin{array}{r}-11.3 \\
+54.6\end{array}$ & 0.60 \\
\hline $48-2,127-129$ & 448.78 & $\begin{array}{r}0.34 \\
\pm 0.03 \\
0.36\end{array}$ & 284.7 & +70.5 & 0.19 & 259.5 & $\begin{array}{l}+57.2 \\
+86.4\end{array}$ & 0.20 & 239.1 & $\begin{array}{l}+38.4 \\
+59.6\end{array}$ & 0.11 & 288.2 & $\begin{array}{r}+54.6 \\
-7.6\end{array}$ & 0.87 \\
\hline $49-3,114-116$ & 459.65 & $\begin{array}{r}0.36 \\
\pm 0.06 \\
1.11\end{array}$ & 252.1 & $\begin{array}{r}+84.5 \\
+65.4\end{array}$ & 0.31 & 94.8 & $\begin{array}{l}+86.4 \\
+53 .\end{array}$ & 0.11 & 111.0 & +36.1 & 0.13 & 230.1 & +22.5 & 1.17 \\
\hline $53-1,118-120$ & 491.34 & $\begin{array}{r}1.11 \\
\pm 0.04 \\
6.26\end{array}$ & 226.2 & +48.2 & 4.60 & 230.3 & +45.1 & 0.21 & 304.0 & - & 0.10 & 年 & - & 0.92 \\
\hline $53-1,118-120$ & 504.19 & $\begin{array}{r}0.20 \\
\pm 0.20 \\
\end{array}$ & 226.2 & +48.2 & 4.60 & 230.0 & 745.1 & - & 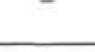 & 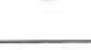 & 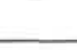 & - & & \\
\hline $54-3,133-135$ & 516.84 & $\begin{array}{l}18.38 \\
\pm 0.06\end{array}$ & 161.6 & +43.2 & 13.89 & 163.5 & +38.6 & 6.73 & 161.4 & +34.4 & 4.14 & 164.7 & +28.2 & 0.35 \\
\hline $55-1,114-116$ & 523.15 & $\begin{array}{l}12.30 \\
\pm 0.21\end{array}$ & 272.8 & +40.6 & 9.01 & 266.0 & +32.0 & 5.77 & 265.3 & +21.2 & 4.12 & 262.1 & +20.9 & 1.62 \\
\hline $55-4,110-112$ & 527.61 & $\begin{array}{r}3.38 \\
\pm 0.22\end{array}$ & 326.2 & +41.1 & 3.31 & 264.0 & -2.3 & 5.54 & 266.7 & -18.3 & 3.36 & 270.3 & -19.4 & 2.10 \\
\hline $56-2,142-144$ & 534.43 & $\begin{array}{r}4.03 \\
\pm 0.24\end{array}$ & 355.5 & +61.9 & 3.80 & 18.9 & +28.8 & 5.03 & 19.4 & -9.9 & 2.92 & 23.4 & -16.8 & 2.24 \\
\hline $57-1,21-23$ & 541.22 & $\begin{array}{r}0.52 \\
\pm 0.06 \\
\end{array}$ & 34.9 & -35.5 & 0.76 & 178.5 & -46.5 & 1.09 & 192.8 & -38.9 & 0.69 & 193.0 & -38.2 & 1.49 \\
\hline $57-3,135-137$ & 545.36 & $\begin{array}{r}7.65 \\
\pm 0.20\end{array}$ & 28.8 & -7.2 & 7.03 & 29.0 & -7.4 & 4.84 & 16.9 & -17.0 & 3.80 & 33.7 & -14.5 & 0.85 \\
\hline $60-6,45-47$ & 577.46 & $\begin{array}{r}0.21 \\
\pm 0.04\end{array}$ & 302.8 & +52.1 & 0.19 & 355.0 & +61.0 & 0.06 & 40.1 & +50.2 & 0.04 & 13.6 & +11.8 & 0.48 \\
\hline $61-2,15-17$ & 580.66 & $\begin{array}{r}0.97 \\
\pm 0.01\end{array}$ & 172.1 & -47.1 & 1.05 & 174.8 & -57.1 & 1.42 & 203.8 & -29.7 & 1.16 & 198.4 & -12.7 & - \\
\hline $61-5,118-120$ & 586.19 & $\begin{array}{r}0.29 \\
+0.02\end{array}$ & 24.9 & +28.8 & 0.16 & 51.2 & +58.2 & 0.05 & 22.3 & +2.8 & 0.04 & 101.9 & +38.4 & 0.74 \\
\hline $62-2,79-81$ & 590.81 & $\begin{array}{r}1.24 \\
\pm 0.03 \\
\end{array}$ & 357.3 & +53.7 & 0.86 & 7.8 & +68.7 & 0.26 & 324.0 & +46.2 & 0.12 & 329.0 & +16.8 & 1.21 \\
\hline $62-4,143-145$ & 594.44 & $\begin{array}{r}4.88 \\
\pm 0.46\end{array}$ & 46.7 & +62.7 & 1.77 & 64.8 & +78.7 & 0.88 & 222.7 & +67.4 & 0.44 & 234.0 & +54.9 & 1.33 \\
\hline $64-4,111-113$ & 613.12 & $\begin{array}{l}13.03 \\
\pm 0.06\end{array}$ & 206.9 & +83.6 & 9.25 & 232.3 & +77.3 & 2.84 & 280.7 & $\begin{array}{r}+77.4 \\
\end{array}$ & 0.90 & 224.9 & +56.2 & 2.77 \\
\hline $66-1,103-105$ & 627.54 & $\begin{array}{r}3.86 \\
\pm 0.32\end{array}$ & 299.3 & +66.2 & 3.75 & 209.7 & +17.5 & 4.36 & 207.7 & -7.0 & 3.18 & 209.6 & -11.9 & 2.41 \\
\hline $66-6,103-105$ & 635.04 & $\begin{array}{r}5.74 \\
\pm 0.15\end{array}$ & 210.6 & +10.0 & 8.38 & 216.3 & -12.6 & 9.05 & 219.0 & -19.6 & 6.10 & 218.8 & -21.8 & 1.59 \\
\hline $67-2,62-64$ & 638.13 & $\begin{array}{r}6.13 \\
\pm 0.46\end{array}$ & 238.3 & +42.2 & 1.80 & 167.0 & +50.1 & 1.64 & 54.8 & -25.5 & 1.32 & 96.6 & -36.3 & 2.38 \\
\hline
\end{tabular}


TABLE 1

Summary of Paleomagnetic Data, Site 397

\begin{tabular}{|c|c|c|c|c|c|c|c|c|c|c|c|c|c|c|}
\hline $\begin{array}{c}\text { Sample } \\
\text { (Interval in cm) }\end{array}$ & $\begin{array}{l}\text { Depth } \\
(\mathrm{m})\end{array}$ & $\begin{array}{l}\text { Int. } \\
(\mu \mathrm{G}) \\
\pm \text { S.D. }\end{array}$ & $\begin{array}{l}\text { NRM } \\
\text { Dec. }\end{array}$ & Inc. & $\begin{array}{l}\text { Int. } \\
(\mu \mathrm{G})\end{array}$ & $\begin{array}{l}50 \mathrm{Oe} \\
\mathrm{Dec} .\end{array}$ & Inc. & $\begin{array}{l}\text { Int. } \\
(\mu \mathrm{G})\end{array}$ & $\begin{array}{c}150 \text { Oe } \\
\text { Dec. }\end{array}$ & Inc. & $\begin{array}{l}\text { Int. } \\
(\mu \mathrm{G})\end{array}$ & $\begin{array}{c}300 \mathrm{Oe} \\
\text { Dec. }\end{array}$ & Inc. & $\begin{array}{l}\text { Susc. } \\
\left(\times 10^{-5}\right. \\
\text { G/Oe })\end{array}$ \\
\hline $1-1,16-18$ & 9.00 & 49.80 & 252.9 & +61.4 & 43.02 & 254.7 & +65.6 & 10.31 & 252.5 & +49.5 & 10.22 & 263.4 & +54.4 & 5.14 \\
\hline $2-1,61-63$ & 9.62 & 0.18 & 106.4 & +6.2 & 9.07 & 107.3 & +4.3 & 5.97 & 104.5 & +8.8 & 3.92 & 106.0 & +15.5 & 1.73 \\
\hline $2-5,80-82$ & 15.81 & 0.13 & 89.5 & +19.2 & 0.10 & 103.3 & -66.2 & - & - & - & 0.04 & 254.7 & +35.6 & 0.27 \\
\hline $4-4,30-32$ & 32.81 & $\begin{array}{r}0.35 \\
\pm 0.02\end{array}$ & 314.0 & +18.2 & 0.50 & 187.9 & +20.2 & 0.11 & 148.1 & -51.0 & 0.07 & 145.3 & -17.5 & 0.10 \\
\hline $5-3,31-33$ & 42.32 & $\begin{array}{r}1.90 \\
+0.25 \\
\end{array}$ & 191.6 & +16.8 & 1.43 & 197.3 & +34.8 & - & - & - & 0.11 & 157.1 & -18.5 & 0.11 \\
\hline $6-1,58-60$ & 47.59 & $\begin{array}{r}1.88 \\
\pm 0.27\end{array}$ & 310.9 & +41.4 & 2.16 & 299.8 & +40.4 & - & - & - & 0.08 & 59.9 & -50.3 & 0.25 \\
\hline $7-6,27-29$ & 64.28 & $\begin{array}{r}3.11 \\
\pm 0.18\end{array}$ & 97.9 & +45.0 & 2.75 & 96.2 & +30.0 & 1.03 & 108.7 & +45.7 & 1.97 & 98.1 & +12.9 & 0.21 \\
\hline $8-4,87-89$ & 71.36 & $\begin{array}{r}1.31 \\
\pm 0.20\end{array}$ & 178.2 & -2.9 & 0.79 & 158.8 & +19.0 & 0.08 & 172.2 & -9.1 & 0.06 & 173.1 & -12.0 & 0.20 \\
\hline $10-3,90-92$ & 88.91 & $\begin{array}{r}0.89 \\
\pm 0.09\end{array}$ & 192.3 & +52.2 & 0.52 & 157.8 & -51.3 & 0.10 & 153.4 & -14.9 & 0.07 & 320.8 & -73.9 & 0.16 \\
\hline $10-6,132-134$ & 92.93 & $\begin{array}{r}0.50 \\
\pm 0.25 \\
\end{array}$ & 53.7 & +23.8 & 0.67 & 255.7 & +9.9 & 0.02 & 160.6 & +74.9 & 0.03 & 119.3 & +46.1 & 0.36 \\
\hline $11-2,49-51$ & 96.50 & $\begin{array}{r}0.88 \\
\pm 0.22\end{array}$ & 239.8 & +46.1 & 0.12 & 274.7 & +60.0 & 0.05 & 313.5 & +47.3 & 0.19 & 95.8 & +18.1 & 0.31 \\
\hline $12-4,14-16$ & 108.15 & $\begin{array}{r}0.96 \\
\pm 0.06\end{array}$ & 347.8 & -52.4 & 0.96 & 344.8 & -43.4 & 0.35 & 62.9 & -71.0 & 0.45 & 119.2 & -51.6 & 0.43 \\
\hline $13-2,129-131$ & 116.50 & $\begin{array}{r}0.17 \\
\pm 0.01\end{array}$ & 108.8 & +32.9 & 0.11 & 174.0 & +15.7 & 0.05 & 154.3 & +26.1 & 0.03 & 274.2 & +74.2 & 0.39 \\
\hline $13-4,36-38$ & 118.37 & $\begin{array}{r}0.44 \\
\pm 0.09\end{array}$ & 206.6 & +65.5 & 0.59 & 237.1 & +66.3 & 0.06 & 173.9 & -28.8 & 0.05 & 122.2 & -36.7 & 0.38 \\
\hline $13-7,32-34$ & 120.83 & $\begin{array}{r}0.76 \\
\pm 0.08 \\
\end{array}$ & 67.4 & +32.9 & 0.46 & 88.9 & +29.1 & 0.05 & 330.0 & +25.3 & 0.02 & 96.9 & -45.4 & 0.40 \\
\hline $\begin{array}{l}15-1,125-127 \\
15-5,140-142\end{array}$ & $\begin{array}{l}133.75 \\
139.91\end{array}$ & $\begin{array}{r}0.53 \\
0.35 \\
\pm 0.17\end{array}$ & $\begin{array}{r}335.4 \\
75.9\end{array}$ & $\begin{array}{l}+80.4 \\
+32.1\end{array}$ & $\begin{array}{l}0.63 \\
0.20\end{array}$ & $\begin{array}{r}58.3 \\
349.4\end{array}$ & $\begin{array}{l}+74.7 \\
+40.2\end{array}$ & $\begin{array}{l}0.08 \\
0.07\end{array}$ & $\begin{array}{r}78.8 \\
214.0\end{array}$ & $\begin{array}{l}+16.0 \\
-15.1\end{array}$ & $\begin{array}{l}0.10 \\
0.05\end{array}$ & $\begin{array}{l}348.5 \\
263.0\end{array}$ & $\begin{array}{l}-17.7 \\
-22.9\end{array}$ & $\begin{array}{l}0.39 \\
0.34\end{array}$ \\
\hline $16-6,20-22$ & 149.71 & $\begin{array}{r}0.40 \\
\pm 0.01\end{array}$ & 315.7 & +62.4 & 0.49 & 268.4 & +54.3 & 0.04 & 69.4 & +19.0 & 0.02 & 78.1 & -58.9 & 0.55 \\
\hline $16-6,71-73$ & 150.22 & $\begin{array}{r}0.25 \\
\pm 0.02\end{array}$ & 315.6 & +13.3 & 0.12 & 226.4 & -35.2 & 0.04 & 126.8 & -63.3 & 0.05 & 144.1 & -62.5 & 0.44 \\
\hline $16-6,112-114$ & 150.62 & $\begin{array}{r}0.31 \\
\pm 0.15 \\
\end{array}$ & 52.6 & -16.2 & 0.16 & 118.8 & -62.6 & 0.03 & 58.5 & -31.0 & 0.05 & 72.9 & -64.9 & 0.51 \\
\hline $17-3,53-55$ & 155.04 & $\begin{array}{r}0.17 \\
\pm 0.05\end{array}$ & 42.1 & +15.0 & 0.18 & 70.1 & -11.4 & 0.10 & 69.2 & -4.2 & 0.09 & 84.8 & -9.6 & 0.46 \\
\hline $17-5,78-80$ & 158.29 & 5 & $\overline{7}$ & $\overline{0}$ & 0.09 & 9.1 & -31.5 & 0.03 & 126.7 & -43.7 & 0.06 & 190.2 & -53.7 & 0.38 \\
\hline $17-6,125-127$ & 160.26 & $\begin{array}{r}0.56 \\
\pm 0.02\end{array}$ & 167.2 & -31.8 & 0.39 & 164.9 & -17.0 & 0.37 & 164.4 & -18.7 & 0.15 & 166.1 & -15.7 & 0.36 \\
\hline $18-4,115-117$ & 166.66 & $\begin{array}{r}0.25 \\
\pm 0.05\end{array}$ & 162.4 & -13.7 & 0.36 & 164.3 & -7.7 & 0.40 & 160.4 & -4.2 & 0.34 & 164.5 & -5.5 & 0.31 \\
\hline $18-6,10-12$ & 169.77 & $\begin{array}{r}0.10 \\
\pm 0.00\end{array}$ & 298.3 & -3.3 & 0.25 & 270.5 & +51.7 & 0.10 & 95.4 & -49.6 & 0.06 & 95.2 & -67.7 & 0.39 \\
\hline $19-6,49-51$ & 178.50 & $\begin{array}{r}0.27 \\
\pm 0.08\end{array}$ & 337.3 & -34.7 & 0.27 & 160.8 & +37.4 & 0.11 & 133.9 & -60.6 & 0.03 & 153.8 & -14.2 & 0.37 \\
\hline $20-2,97-99$ & 182.48 & $\begin{array}{r}0.20 \\
\pm 0.14\end{array}$ & 42.0 & -1.5 & 0.10 & 0.6 & -33.4 & 0.07 & 7.2 & -56.5 & 0.06 & 353.4 & -47.5 & 0.12 \\
\hline $20-5,116-118$ & 187.17 & $\begin{array}{r}0.20 \\
\pm 0.07\end{array}$ & 19.1 & -35.7 & 0.16 & 291.0 & -15.0 & 0.07 & 162.9 & -36.9 & 0.04 & 317.0 & -61.5 & 0.50 \\
\hline $21-3,135-137$ & 193.86 & $\begin{array}{r}0.25 \\
\pm 0.09\end{array}$ & 290.0 & -9.0 & 0.27 & 245.8 & -54.5 & 0.08 & 268.3 & -41.4 & 0.10 & 262.4 & -61.7 & 0.31 \\
\hline $21-4,58-60$ & 194.59 & $\begin{array}{r}0.19 \\
\pm 0.05 \\
\end{array}$ & 354.0 & -35.6 & 0.06 & 53.5 & -14.4 & 0.10 & 116.4 & -35.6 & 0.06 & 118.7 & -31.6 & 0.38 \\
\hline $22-2,56-58$ & 201.06 & 0.17 & 32.7 & -3.8 & 0.09 & 277.0 & +8.1 & 0.06 & 54.7 & -16.5 & 0.04 & 45.4 & -41.4 & 0.35 \\
\hline $22-6,29-31$ & 206.80 & $\begin{array}{r}0.13 \\
\pm 0.04\end{array}$ & 44.7 & +24.4 & 0.07 & 27.2 & -36.5 & 0.07 & 48.9 & -49.4 & 0.05 & 135.4 & -74.7 & 0.20 \\
\hline $23-2,114-116$ & 211.15 & $\begin{array}{r}0.16 \\
\pm 0.01\end{array}$ & 322.0 & +23.6 & 0.05 & 299.2 & +43.4 & 0.04 & 54.6 & +28.3 & 0.06 & 41.7 & -27.4 & 0.30 \\
\hline $23-5,38-40$ & 214.89 & $\begin{array}{r}0.14 \\
\pm 0.07\end{array}$ & 354.3 & +39.9 & 0.09 & 59.8 & +40.5 & 0.10 & 41.4 & +21.0 & 0.05 & 70.2 & +8.6 & 0.40 \\
\hline $24-1,29-31$ & 218.30 & $\begin{array}{r}0.15 \\
\pm 0.10 \\
\end{array}$ & 281.3 & +33.5 & 0.05 & 241.2 & +15.7 & 0.05 & 336.9 & -0.2 & 0.14 & 131.1 & -31.1 & 0.35 \\
\hline $24-4,90-92$ & 223.41 & $\begin{array}{r}0.13 \\
\pm 0.03\end{array}$ & 299.1 & +15.0 & 0.17 & 271.8 & +15.7 & 0.04 & 315.8 & -37.2 & 0.01 & 355.3 & -55.6 & 0.62 \\
\hline $24-7,6-8$ & 227.07 & $\begin{array}{r}0.10 \\
\pm 0.02\end{array}$ & 344.9 & -20.1 & 0.06 & 339.7 & -59.7 & 0.10 & 318.2 & -15.3 & 0.03 & 296.1 & -40.3 & - \\
\hline $25-5,35-37$ & 233.86 & $\begin{array}{r}0.18 \\
\pm 0.01\end{array}$ & 351.8 & 45.8 & 0.05 & 106.3 & +28.3 & 0.16 & 76.8 & -12.1 & 0.11 & 80.6 & -29.0 & 0.20 \\
\hline $26-1,36-38$ & 237.37 & $\begin{array}{r}0.13 \\
\pm 0.05\end{array}$ & 67.8 & -0.2 & 0.14 & 216.6 & +55.9 & 0.12 & 292.8 & +24.0 & 0.12 & 284.1 & +29.4 & 0.36 \\
\hline $27-4,87-89$ & 251.88 & $\begin{array}{r}0.26 \\
\pm 0.01 \\
\end{array}$ & 337.2 & +59.4 & 0.13 & 299.8 & -44.5 & 0.03 & 102.6 & -53.1 & 0.03 & 51.8 & -16.0 & 0.36 \\
\hline
\end{tabular}


TABLE 1 - Continued

\begin{tabular}{|c|c|c|c|c|c|c|c|c|c|c|c|c|c|c|}
\hline $\begin{array}{c}\text { Sample } \\
\text { (Interval in } \mathrm{cm} \text { ) }\end{array}$ & $\begin{array}{l}\text { Depth } \\
\text { (m) }\end{array}$ & $\begin{array}{c}\text { Int. } \\
(\mu \mathrm{G}) \\
\pm \text { S.D. }\end{array}$ & $\begin{array}{l}\text { NRM } \\
\text { Dec. }\end{array}$ & Inc. & $\begin{array}{l}\text { Int. } \\
(\mu \mathrm{G})\end{array}$ & $\begin{array}{l}50 \mathrm{Oe} \\
\mathrm{Dec} .\end{array}$ & Inc. & $\begin{array}{l}\text { Int. } \\
(\mu \mathrm{G})\end{array}$ & $\begin{array}{c}150 \text { Oe } \\
\text { Dec. }\end{array}$ & Inc. & $\begin{array}{l}\text { Int. } \\
(\mu \mathrm{G})\end{array}$ & $\begin{array}{c}300 \mathrm{Oe} \\
\text { Dec. }\end{array}$ & Inc. & $\begin{array}{c}\text { Susc. } \\
\left(\times 10^{-5}\right. \\
\text { G/Oe })\end{array}$ \\
\hline $87-1,59-61$ & 826.60 & $\begin{array}{r}0.60 \\
\pm 0.19\end{array}$ & 24.9 & +33.4 & 0.46 & 308.7 & -23.9 & - & - & - & - & - & - & 0.11 \\
\hline $87-2,84-86$ & 828.35 & $\begin{array}{r}0.60 \\
\pm 0.07\end{array}$ & 58.3 & +66.9 & 0.24 & 30.8 & +43.6 & 0.28 & 1.0 & -4.2 & 0.03 & 10.3 & -7.2 & 1.13 \\
\hline $88-4,29-31$ & 840.30 & $\begin{array}{r}0.12 \\
\pm 0.07\end{array}$ & 345.2 & +45.6 & 0.32 & 157.9 & +42.4 & 0.13 & 7.2 & +16.4 & 0.04 & 134.8 & +24.6 & 1.17 \\
\hline $89-3,79-81$ & 848.80 & $\begin{array}{r}0.33 \\
+0.02\end{array}$ & 244.9 & +45.0 & 0.28 & 230.6 & +45.8 & 0.12 & 197.2 & -1.9 & 0.03 & 271.8 & +10.0 & 1.35 \\
\hline $95-4,8-10$ & 906.59 & $\begin{array}{r}2.47 \\
+0.23\end{array}$ & 293.2 & -32.8 & 1.58 & 283.4 & -7.8 & 0.09 & 344.3 & -21.3 & 0.05 & 260.4 & -45.5 & 0.92 \\
\hline \multicolumn{15}{|l|}{ Hole 397A } \\
\hline $6-1,28-30$ & 963.79 & $\begin{array}{r}0.14 \\
=0.05\end{array}$ & 247.2 & +85.0 & 0.11 & 255.0 & +4.3 & - & - & - & - & - & - & 0.72 \\
\hline $7-1,27-29$ & 982.78 & $\begin{array}{r}5.21 \\
\pm 0.03 \\
\end{array}$ & 359.2 & +31.2 & 2.21 & 359.6 & +36.9 & 0.89 & 357.5 & +21.9 & 0.19 & 331.6 & +43.5 & 2.56 \\
\hline $10-2,76-78$ & 1013.27 & $\begin{array}{r}0.03 \\
\pm 0.02\end{array}$ & 3.1 & -9.8 & 0.02 & 178.7 & +28.7 & 0.01 & 276.3 & +63.8 & 0.03 & 321.9 & +3.3 & 0.38 \\
\hline $11-3,99-101$ & 1024.50 & $\begin{array}{r}0.10 \\
\pm 0.05\end{array}$ & 214.4 & +67.9 & 0.12 & 251.9 & +72.2 & - & - & - & - & - & - & - \\
\hline $11-6,129-132$ & 1029.30 & $\begin{array}{r}0.22 \\
\pm 0.04\end{array}$ & 37.1 & +88.1 & 0.17 & 319.9 & +75.7 & 0.08 & 216.8 & +77.4 & 0.04 & 234.0 & +2.4 & 0.53 \\
\hline $12-1,109-112$ & 1031.10 & $\begin{array}{r}0.10 \\
\pm 0.02\end{array}$ & 177.5 & +20.1 & 0.12 & 198.0 & +22.4 & 0.05 & 134.6 & +71.0 & 0.06 & 99.8 & +38.2 & 0.80 \\
\hline $13-2,106-108$ & 1042.07 & $\begin{array}{r}0.15 \\
\pm 0.04 \\
\end{array}$ & 332.8 & +23.7 & 0.13 & 341.2 & +43.7 & 0.04 & 329.7 & +27.4 & 0.05 & 328.8 & +31.8 & 0.63 \\
\hline $14-3,31-33$ & 1052.32 & $\begin{array}{r}0.12 \\
\pm 0.01\end{array}$ & 24.4 & +69.7 & 0.09 & 133.0 & +73.4 & - & - & - & - & - & - & 0.70 \\
\hline $15-3,98-100$ & 1062.49 & $\begin{array}{r}0.12 \\
\pm 0.01\end{array}$ & 277.8 & +60.4 & 0.17 & 296.4 & +61.7 & 0.05 & 284.0 & +27.4 & 0.03 & 280.7 & +52.6 & 0.87 \\
\hline $15-3,119-121$ & 1062.70 & $\begin{array}{r}0.15 \\
\pm 0.10\end{array}$ & 352.5 & +40.3 & 0.10 & 88.0 & +24.9 & 0.01 & 289.6 & +20.8 & 0.02 & 294.1 & +72.1 & 0.65 \\
\hline $15-4,42-44$ & 1063.43 & $\begin{array}{r}0.20 \\
\pm 0.02\end{array}$ & 50.0 & +61.3 & 0.15 & 84.0 & +52.4 & 0.05 & 9.4 & +49.3 & 0.02 & 17.3 & +30.9 & 0.89 \\
\hline $16-2,81-83$ & 1070.30 & $\begin{array}{r}0.22 \\
\pm 0.04 \\
\end{array}$ & 351.5 & +35.8 & 0.15 & 315.9 & +63.4 & 0.04 & 310.7 & +11.3 & 0.04 & 298.3 & -43.8 & 0.49 \\
\hline $17-2,19-21$ & 1079.20 & $\begin{array}{r}0.09 \\
\pm 0.01\end{array}$ & 309.7 & +30.1 & 0.08 & 289.0 & +49.6 & 0.03 & 334.2 & +11.4 & 0.03 & 359.9 & -28.2 & 0.48 \\
\hline $19-2,115-117$ & 1099.16 & $\begin{array}{r}0.81 \\
\pm 0.01\end{array}$ & 259.3 & +53.6 & 0.62 & 244.6 & +47.5 & 0.25 & 235.0 & +35.5 & 0.18 & 234.7 & +19.2 & 1.29 \\
\hline $20-2,96-98$ & 1108.47 & $\begin{array}{r}0.13 \\
\pm 0.04\end{array}$ & 98.6 & +35.2 & 0.12 & 89.0 & +56.2 & 0.04 & 78.1 & +18.2 & 0.02 & 153.4 & +5.0 & 1.99 \\
\hline $21-1,4-6$ & 1115.55 & $\begin{array}{r}0.30 \\
\pm 0.02\end{array}$ & 358.8 & +42.3 & 0.28 & 340.0 & +34.9 & 0.17 & 325.6 & +18.8 & 0.07 & 338.2 & +25.2 & 1.46 \\
\hline $22-2,56-58$ & 1136.57 & $\begin{array}{r}1.05 \\
\pm 0.06 \\
\end{array}$ & 3.6 & +5.3 & - & - & - & 0.28 & 348.6 & +34.8 & 0.12 & 347.6 & +35.0 & 1.40 \\
\hline $22-2,120-122$ & 1137.21 & $\begin{array}{r}0.12 \\
\pm 0.03\end{array}$ & 239.3 & +56.2 & - & - & - & 0.09 & 261.8 & +35.1 & 0.05 & 246.8 & +21.7 & - \\
\hline $23-3,145-147$ & 1157.96 & $\begin{array}{r}0.17 \\
\pm 0.02\end{array}$ & 21.5 & +27.0 & 0.14 & 30.0 & +33.5 & 0.10 & 60.2 & +29.9 & 0.11 & 78.6 & +15.2 & - \\
\hline $23-4,134-136$ & 1159.35 & $\begin{array}{r}0.17 \\
\pm 0.03\end{array}$ & 309.3 & +43.2 & 0.12 & 305.6 & +9.4 & 0.07 & 329.0 & +34.3 & 0.03 & 343.5 & +35.9 & 1.07 \\
\hline $24-1,137-139$ & 1173.88 & $\begin{array}{r}0.46 \\
\pm 0.02\end{array}$ & 339.4 & +30.1 & - & - & - & 0.13 & 252.8 & +49.2 & 0.08 & 19.6 & +85.7 & 0.28 \\
\hline $24-2,55-57$ & 1174.56 & $\begin{array}{r}0.19 \\
+0.14 \\
\end{array}$ & 206.9 & +51.8 & 0.25 & 217.9 & +56.3 & - & - & - & - & - & - & 0.33 \\
\hline $27-1,106-109$ & 1230.57 & $\begin{array}{r}0.38 \\
\pm 0.03\end{array}$ & 16.6 & +40.9 & 0.28 & 14.2 & +46.8 & 0.12 & 86.2 & +54.1 & 0.06 & 62.3 & +55.7 & 0.99 \\
\hline $28-1,136-138$ & 1240.57 & $\begin{array}{r}0.44 \\
\pm 0.01\end{array}$ & 193.6 & +67.6 & 0.24 & 208.5 & +53.7 & 0.18 & 219.1 & +43.6 & 0.10 & 215.8 & +45.9 & 1.07 \\
\hline $28-2,41-43$ & 1240.92 & $\begin{array}{r}0.19 \\
\pm 0.15\end{array}$ & 108.9 & +74.0 & 0.30 & 311.9 & +63.7 & - & - & - & - & - & - & 0.47 \\
\hline $28-2,45-47$ & 1240.95 & $\begin{array}{r}0.24 \\
\pm 0.08\end{array}$ & 36.3 & +26.2 & 0.17 & 71.0 & -14.3 & 0.02 & 167.4 & +20.5 & 0.04 & 68.6 & -2.2 & 0.43 \\
\hline $28-2,110-112$ & 1241.61 & $\begin{array}{r}1.75 \\
\pm 0.15 \\
\end{array}$ & 150.3 & +46.9 & 1.04 & 152.5 & +47.3 & 0.12 & 148.3 & +53.7 & 0.07 & 107.6 & +64.5 & 1.51 \\
\hline $30-1,67-69$ & 1258.68 & $\begin{array}{r}0.76 \\
\pm 0.02\end{array}$ & 184.9 & +68.0 & 0.54 & 227.3 & +77.1 & 0.16 & 304.6 & +64.3 & 0.08 & 252.8 & +71.5 & 1.09 \\
\hline $32-2,132-134$ & 1279.83 & $\begin{array}{r}0.16 \\
\pm 0.07\end{array}$ & 313.6 & -23.5 & 0.12 & 183.1 & +61.1 & - & - & - & - & - & - & 0.56 \\
\hline $34-1,12-14$ & 1296.13 & $\begin{array}{r}0.35 \\
\pm 0.03\end{array}$ & 99.9 & +58.4 & 0.26 & 55.0 & +79.9 & 0.10 & 92.9 & +68.8 & 0.07 & 170.0 & +73.0 & 1.06 \\
\hline $34-2,95-96$ & 1298.45 & $\begin{array}{r}0.94 \\
\pm 0.03\end{array}$ & 348.8 & +41.4 & 0.86 & 354.1 & +40.1 & - & - & - & 0.30 & 67.4 & +21.4 & 1.59 \\
\hline $35-2,75-78$ & 1307.26 & $\begin{array}{r}2.70 \\
\pm 0.10 \\
\end{array}$ & 65.3 & +24.2 & 2.67 & 81.6 & +24.9 & 2.91 & 99.2 & -4.0 & 3.92 & 106.4 & -23.6 & 2.47 \\
\hline $35-3,128-131$ & 1309.29 & $\begin{array}{r}3.60 \\
\pm 0.36 \\
\end{array}$ & 245.6 & -23.4 & - & - & - & - & - & - & 5.64 & 225.4 & -17.5 & 2.72 \\
\hline
\end{tabular}


TABLE 1 - Continued

\begin{tabular}{|c|c|c|c|c|c|c|c|c|c|c|c|c|c|c|}
\hline $\begin{array}{c}\text { Sample } \\
\text { (Interval in } \mathrm{cm} \text { ) }\end{array}$ & $\begin{array}{l}\text { Depth } \\
(\mathrm{m})\end{array}$ & $\begin{aligned} & \text { Int. } \\
& (\mu \mathrm{G}) \\
\pm & \mathrm{S} . \mathrm{D} .\end{aligned}$ & $\begin{array}{l}\text { NRM } \\
\text { Dec. }\end{array}$ & Inc. & $\begin{array}{l}\text { Int. } \\
(\mu \mathrm{G})\end{array}$ & $\begin{array}{l}50 \mathrm{Oe} \\
\text { Dec. }\end{array}$ & Inc. & $\begin{array}{l}\text { Int. } \\
(\mu \mathrm{G})\end{array}$ & $\begin{array}{c}150 \mathrm{Oe} \\
\text { Dec. }\end{array}$ & Inc. & Int. & $\begin{array}{c}300 \mathrm{Oe} \\
\text { Dec. }\end{array}$ & Ine. & $\begin{array}{c}\text { Susc. } \\
\left(\times 10^{-5}\right. \\
\text { G/Oe })\end{array}$ \\
\hline $37-2,136-139$ & 1317.46 & $\begin{array}{r}1.19 \\
\pm 0.04\end{array}$ & 243.9 & +56.8 & - & - & - & - & - & - & 0.25 & 229.7 & +42.2 & 1.38 \\
\hline $39-1,95-97$ & 1334.96 & $\begin{array}{r}1.56 \\
\pm 0.25\end{array}$ & 134.1 & +68.7 & 1.12 & 142.3 & +66.4 & - & - & - & 0.39 & 136.8 & +39.7 & 0.95 \\
\hline $39-2,34-36$ & 1335.85 & $\begin{array}{r}1.07 \\
+0.01\end{array}$ & 109.4 & +63.0 & - & - & - & - & - & - & 0.40 & 97.2 & +63.3 & 0.97 \\
\hline $40-1,145-147$ & 1344.96 & $\begin{array}{l}22.56 \\
\pm 0.44\end{array}$ & 105.5 & +38.0 & 22.22 & 109.0 & +36.9 & 20.62 & 111.0 & +34.0 & 16.02 & 113.7 & +31.8 & 3.53 \\
\hline $40-2,124-65$ & 1346.25 & $\begin{array}{r}8.40 \\
+0.01\end{array}$ & 240.4 & +57.6 & 7.73 & 241.5 & +53.3 & - & - & - & 4.38 & 253.0 & +49.7 & 3.42 \\
\hline $41-2,139-141$ & 1355.90 & $\begin{array}{r}1.51 \\
\pm 0.42\end{array}$ & 292.4 & +27.9 & - & - & - & - & - & - & 0.93 & 213.4 & +41.3 & 1.27 \\
\hline $42-1,60-62$ & 1363.13 & $\begin{array}{l}10.86 \\
\pm 0.07\end{array}$ & 13.5 & +20.4 & - & - & - & - & - & - & 4.10 & 348.3 & +40.4 & 1.98 \\
\hline $43-2,35-37$ & 1373.86 & $\begin{array}{r}43.88 \\
\pm 0.28\end{array}$ & 276.5 & +47.8 & - & - & - & - & - & - & 31.73 & 262.6 & +43.4 & 3.59 \\
\hline $46-1,126-128$ & 1392.27 & $\begin{array}{l}24.23 \\
\pm 1.51\end{array}$ & 24.9 & +20.9 & - & - & - & - & - & - & 18.21 & 19.6 & +25.6 & 2.18 \\
\hline $46-3,30-32$ & 1394.31 & $\begin{array}{r}1.55 \\
\pm 0.27\end{array}$ & 7.2 & +48.2 & - & - & - & - & - & - & 0.48 & 20.4 & +31.2 & - \\
\hline $47-1,108-110$ & 1401.59 & $\begin{array}{l}10.55 \\
\pm 0.14\end{array}$ & 30.6 & -2.1 & 9.25 & 32.5 & +8.7 & - & - & - & 4.84 & 31.6 & +8.1 & 2.58 \\
\hline $47-4,11-14$ & 1405.12 & $\begin{array}{r}2.10 \\
\pm 0.14\end{array}$ & 56.6 & +23.7 & - & - & - & - & - & - & 2.30 & 52.0 & +27.6 & 2.41 \\
\hline $48-2,38-40$ & 1411.89 & $\begin{array}{l}37.46 \\
\pm 0.72\end{array}$ & 181.4 & +51.3 & - & - & - & - & - & - & 27.03 & 172.4 & +48.5 & 3.31 \\
\hline $50-2,67-70$ & 1431.18 & $\begin{array}{r}22.83 \\
+0.67\end{array}$ & 273.4 & +57.1 & - & - & - & - & - & - & 16.82 & 252.4 & +50.1 & 4.69 \\
\hline $50-4,80-84$ & 1434.32 & $\begin{array}{l}25.11 \\
\pm 0.01\end{array}$ & 228.1 & +46.7 & 26.82 & 232.7 & +50.2 & 25.59 & 233.2 & +47.7 & 21.14 & 230.8 & +48.6 & 4.55 \\
\hline $51-1,140-142$ & 1439.91 & $\begin{array}{l}49.31 \\
\pm 0.20\end{array}$ & 185.7 & +48.1 & - & - & - & - & - & - & 40.86 & 180.9 & +43.3 & 5.80 \\
\hline $51-6,85-86$ & 1446.86 & $\begin{array}{l}15,40 \\
\pm 0.09\end{array}$ & 139.3 & +48.6 & - & - & - & - & - & - & 10.53 & 143.5 & +42.7 & 2.39 \\
\hline $52-1,98-103$ & 1448.99 & $\begin{array}{l}25.46 \\
\pm 0.21\end{array}$ & 107.2 & +55.9 & - & - & - & - & - & - & 14.89 & 109.2 & +44.8 & 3.05 \\
\hline $52-3,73-75$ & 1451.76 & $\begin{array}{l}35.90 \\
\pm 0.25\end{array}$ & 334.5 & +54.1 & - & - & - & - & - & - & 29.27 & 328.4 & +56.2 & 4.56 \\
\hline
\end{tabular}

tions, indicative of acquisition of magnetization during epochs of normal polarity. Alternating field demagnetization up to peak fields of 600 Oe for three pilot samples (Figure 4) suggests that these sediments possess a moderately stable remanence, subsequently confirmed by demagnetization of the remaining samples at a single peak field value of 300 Oe (Table 1). Negative inclinations are encountered only in Core 397A-35, which is located close to the hiatus. The removal of an overprinted soft normal polarity component is demonstrated during progressive demagnetization of Sample 397A-35-2, 75-78 cm (Figure 4) by both directional change and the apparent increase in intensity shown by the normalized intensity decay curve.

\section{Magnetic Stratigraphy}

Assignment and correlation of the observed polarity reversals inferred from the paleomagnetic data at Site 397 constitute a major objective of this paper. Figure 5 shows a composite plot of the downhole variation in magnetic inclination for shipboard samples from Holes 397 and 397A after magnetic cleaning. The polarity stratigraphy is derived from these data as shown in the figure. This is done by assuming that negative inclinations correspond to reversed polarities and positive inclination to normal polarities. Such a convention was adopted in earlier DSDP sedimentary paleomagnetic studies (Henry and Opdyke, 1970; Hamilton, 1974; and Ade-Hall and Johnson, 1976). Unfortunately, lack of azimuthal orientation of the cores themselves precludes the use of $160^{\circ}-180^{\circ}$ remanence declination change as confirmatory evidence for polarity reversal. Nevertheless, as Site 397 is located at a moderate latitude, inclination sense can be used fairly reliably as a polarity index. Ambiguity may occasionally arise due to inadequacies of sample coverage or possibly due to effects of localized magnetic excursions, but this is unlikely to obscure entirely the inferred magnetic stratigraphy. Biostratigraphical zonal assignments determined from shipboard and subsequent shore-based paleontological study are shown also in Figure 5.

\section{Quaternary and Neogene Sediments}

Correlation of the magnetic stratigraphy to established geomagnetic time scales can then be attempted. A number of varied compilations of magnetic polarity time scales for the Cenozoic and Mesozoic, or parts of these eras, have been published recently (Ryan et al. 1974; Tarling and Mitchell, 1976; van Hinte, 1976; La Brecque et al., 1977; and Berggren et al., 1978). At Site 397 , the upper 1300 meters of the sediment column is dated as Neogene to Quaternary in age so that correlation of the polarity sequence has the limits from Brunhes Epoch to possibly Epoch 20/21 times. The absolute 

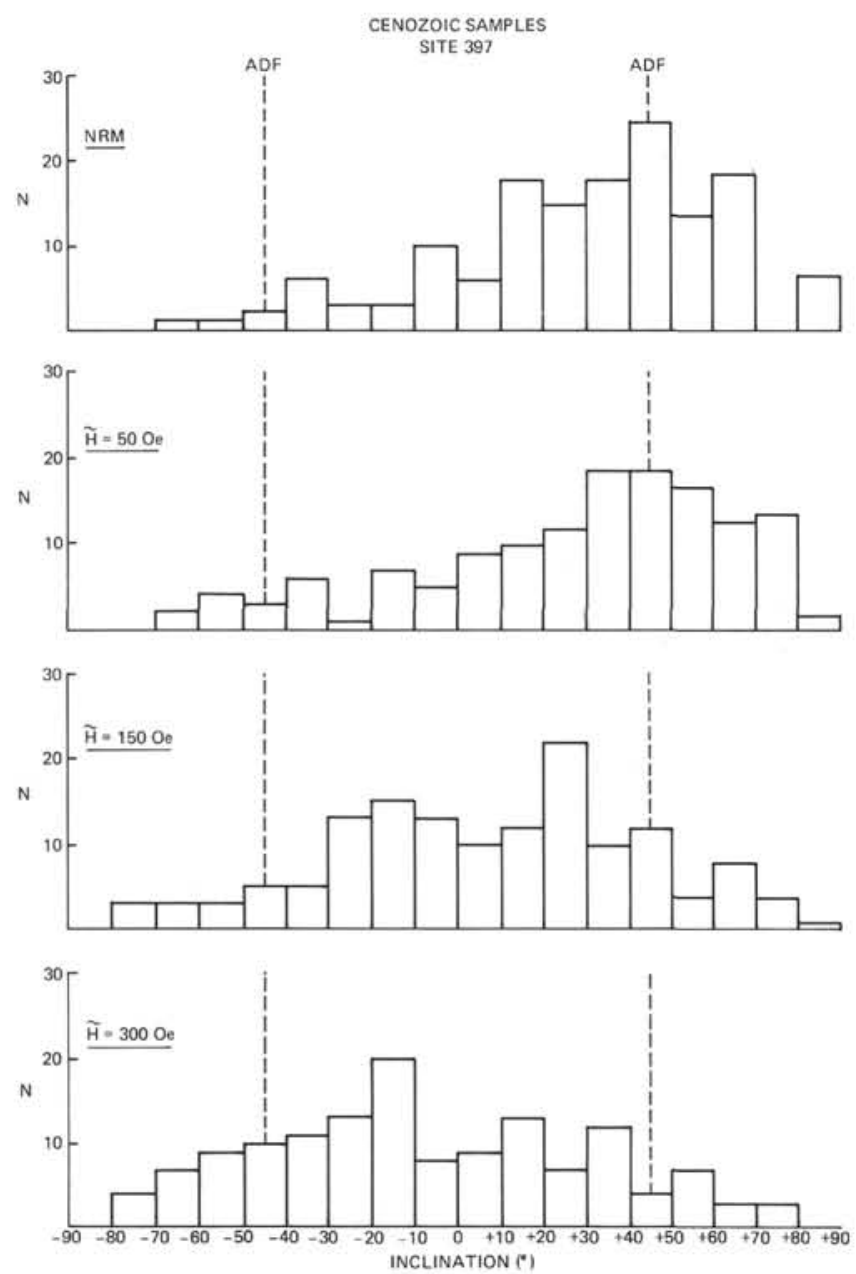

Figure 3. Histograms of remanence inclination values for Cenozoic samples. Dashed vertical line labeled ADF corresponds to present-day axial geocentric dipole field inclination for Site 397.

chronology of these magnetic polarity epochs due to several different authors is shown in Figure 6, together with a suggested correlation of the Site 397 polarities. This correlation is constrained by biostratigraphical assignments.

It is possible to deduce precise sediment accumulation rates from the magnetic stratigraphy when referred to an absolute chronology. This is done in Figure 7 using a calibration to the Ryan et al. (1974) geomagnetic time scale. Despite some degree of uncertainty in the correlation for some parts of the sediment record, several interesting features emerge. Obviously for the upper continental rise of the northwest African continental margin, high sedimentation rates prevailed during the early Miocene, late Pliocene, and Quaternary. The hiatus in the sedimentary record dated as middle Miocene, which corresponds approximately to the $\mathrm{D}_{2}$ seismic reflector (see Site 397 Report) occurred between Epoch 14 and possibly Epoch 11 according to the correlated magnetic stratigraphy. Higher in the record, the episode of slumping in the late Miocene can be dated within Epoch 8 times.
One discovery from the magnetic stratigraphy is the recognition of a possible hiatus in the early Pleistocene, i.e., in the upper Matuyama Epoch. Evidence for this is deduced as explained below. Above the top of the Gauss Epoch, which is located at 208 meters sub-bottom, an interval of reversed polarity to 160 meters is assigned to the lower Matuyama Epoch. A short normal event intervenes before the onset of a normal polarity event which is recognized here as the Olduvai event. Biozonations support such an assigment as this normal event is associated with the first evolutionary appearance of Globorotalia truncatulinoides (N 22) and the extinction of Discoaster brouweri (NN 18). Implications of a possible "split" Olduvai event will not be
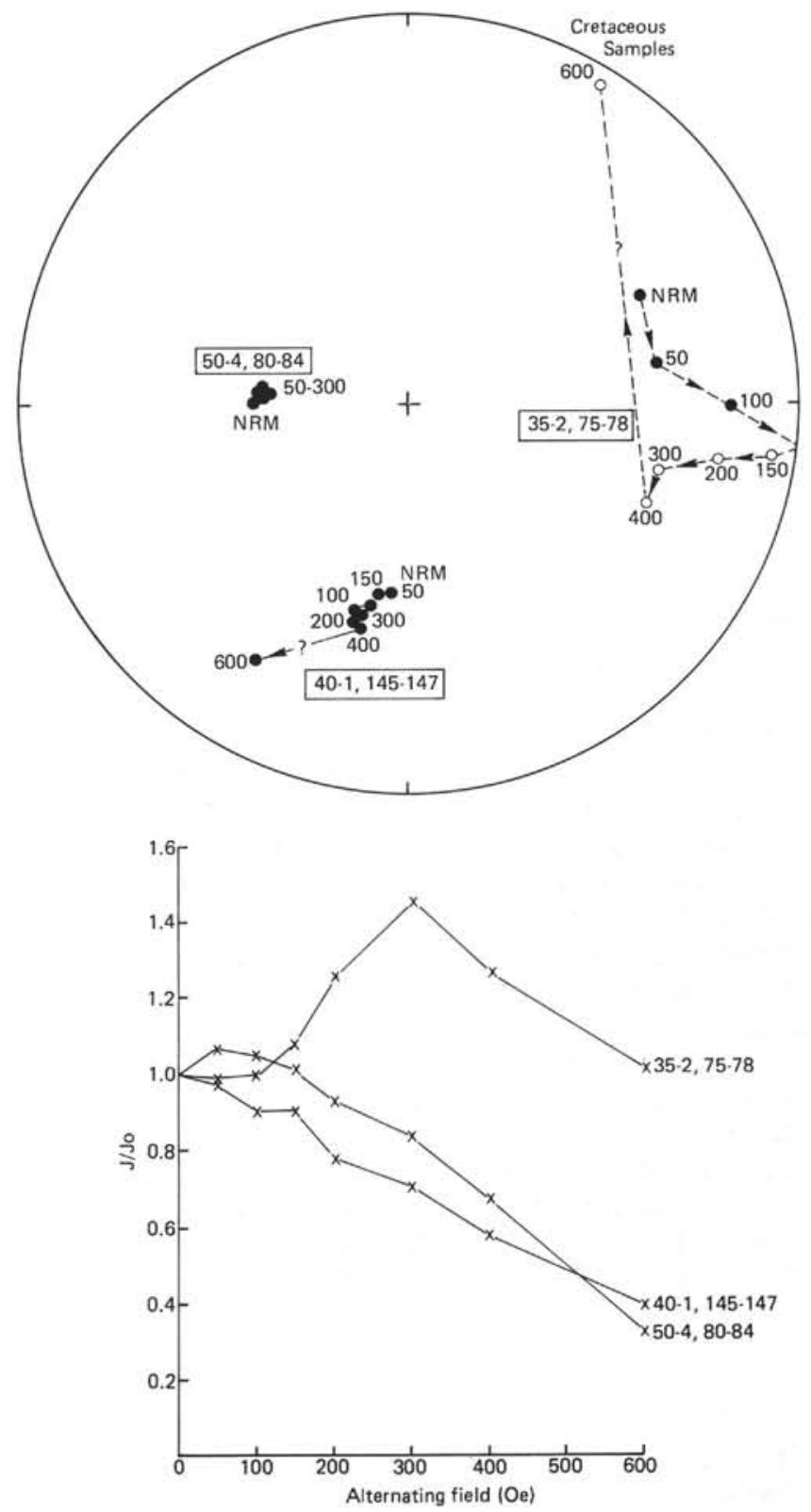

Figure 4. Progressive alternating field demagnetization of selected samples from the Early Cretaceous sediments of Site 397 and associated normalized intensity decay curves. Symbols are in Figure 2. 


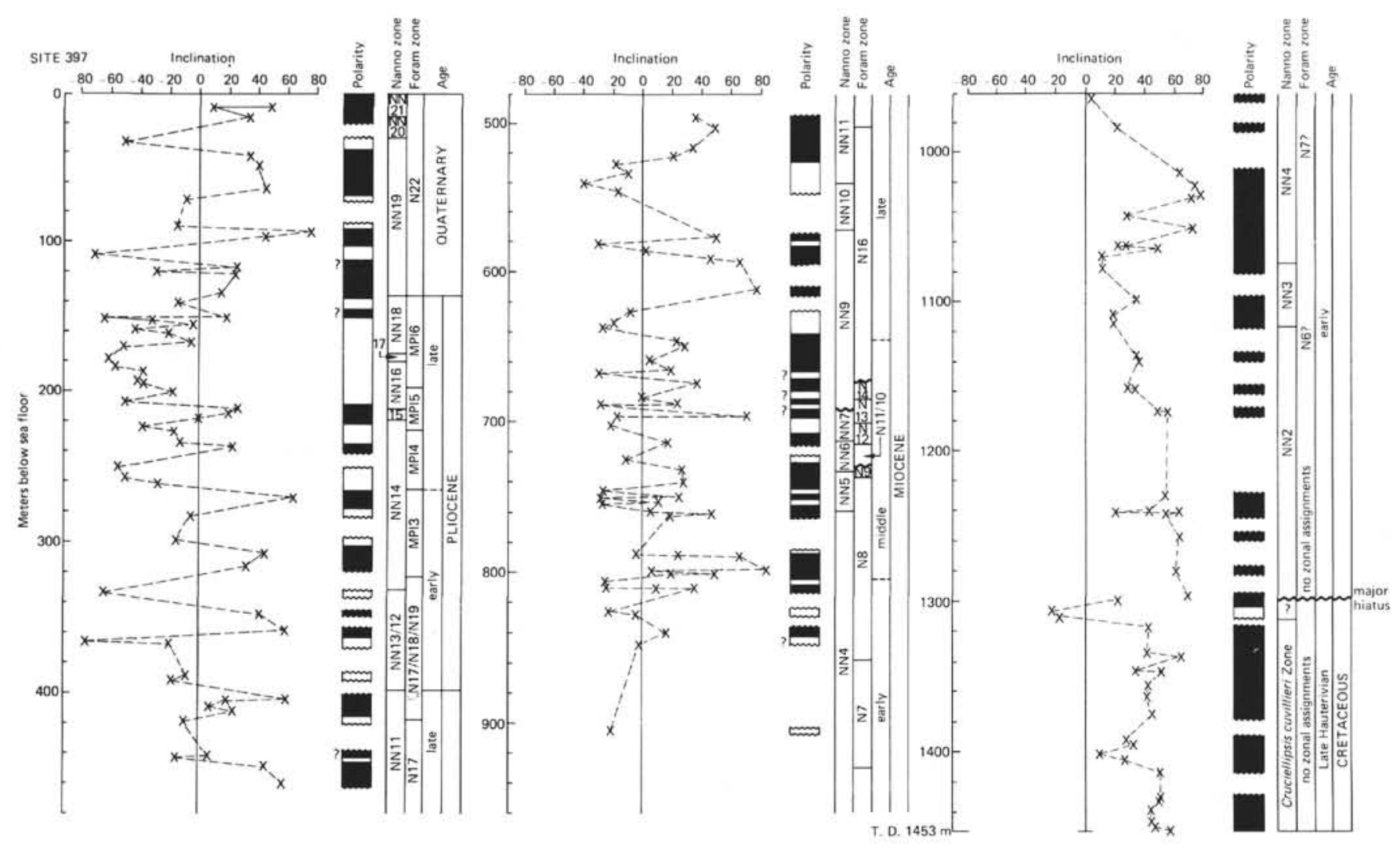

Figure 5. Downhole remanence inclination and inferred magnetic polarity at Site 397.

discussed here, but it is noted that the data from Site 397 record this. However, Briskin and Berggren's (1975) usage is followed to identify the longer normal event as the Olduvai.

A very thin reversed zone, less than 10 meters in thickness, occurs following the Olduvai event. The succeeding normal polarity event located between 92 and 103 meters is tentatively recognized as the Jaramillo event. Such an assignment immediately raises the question of whether the preceding thin reversed interval contains an important hiatus in sedimentation which is not recognized biostratigraphically, although there is evidence of reworking of older faunas in Core 12 (104-113.5 m sub-bottom). An alternative interpretation is that a dramatic reduction in sedimentation rate occurred immediately following the Olduvai event. High accumulation rates recommenced during Jaramillo times and continued through the Brunhes Epoch at Site 397.

It is difficult to assess the validity of the supposed hiatus on the basis of micropaleontological evidence as no faunal boundaries are recognized in this critical interval of the sedimentary record. A re-examination of the nannofossils using the refined Quaternary zonation of Gartner (1977) may provide a critical test of the presence of a hiatus. There remains the possibility that the initial assignment of the upper normal polarity event within the Matuyama Epoch at this site to the Jaramillo event is incorrect, but this appears unlikely on the basis of the inferred sedimentation rate curve. If this event is identified instead as part of the Olduvai event sequence rather than the Jaramillo event, then by inference the Jaramillo event must be absent at this site if the Brunhes/Matuyama boundary is correctly placed. Again, such an interpretation requires a hiatus to be present. It is interesting to note other confirmatory evidence for the hiatus is provided from the recognition of an important change in acoustic impedance recorded from the physical properties data, as well as the presence of a strong reflecting horizon at approximately 100 meters sub-bottom.

Sixty-eight meters of sediment have accumulated during the Brunhes Epoch at Site 397. Unfortunately, the resolution of a detailed magnetic stratigraphy through the Brunhes sequence cannot be achieved because of drilling disturbance in the upper cores. Sampling is necessarily very scant in this interval. Despite this limitation, the data indicate the existence of a possible reversed polarity interval at approximately 25 to 55 meters sub-bottom. The reversed interval may be significant as it occurs close to the placement of the nannofossil zone boundary NN 19/NN 20 at 30 meters. This paleontological boundary corresponds to the extinction of Pseudoemiliana lacunosa during cold Stage 12 of the Pleistocene, approximately 400,000 years B.P. (Gartner and Emiliani, 1976).

The occurrence of globally recognized reversed polarity events within the Brunhes Normal Epoch is still the subject of debate. Notably, attention has focussed on the Laschamps event and the Blake event and their possible correlatives, but these are considerably 


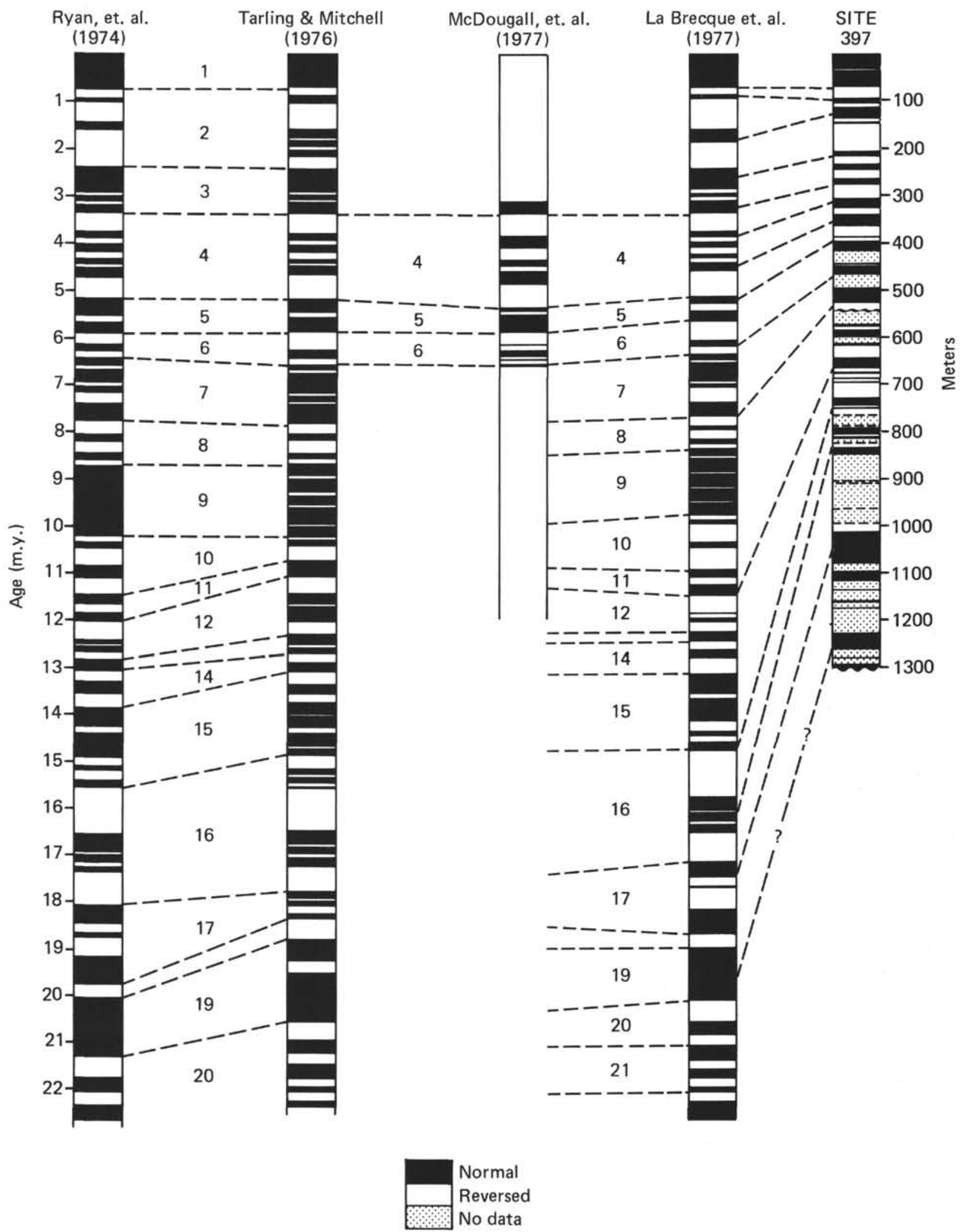

Figure 6. Comparison of geomagnetic polarity time scales for the Quaternary and Neogene and infrared magnetic stratigraphy at Site 397. Numerals between dashed lines refer to epoch designation. 


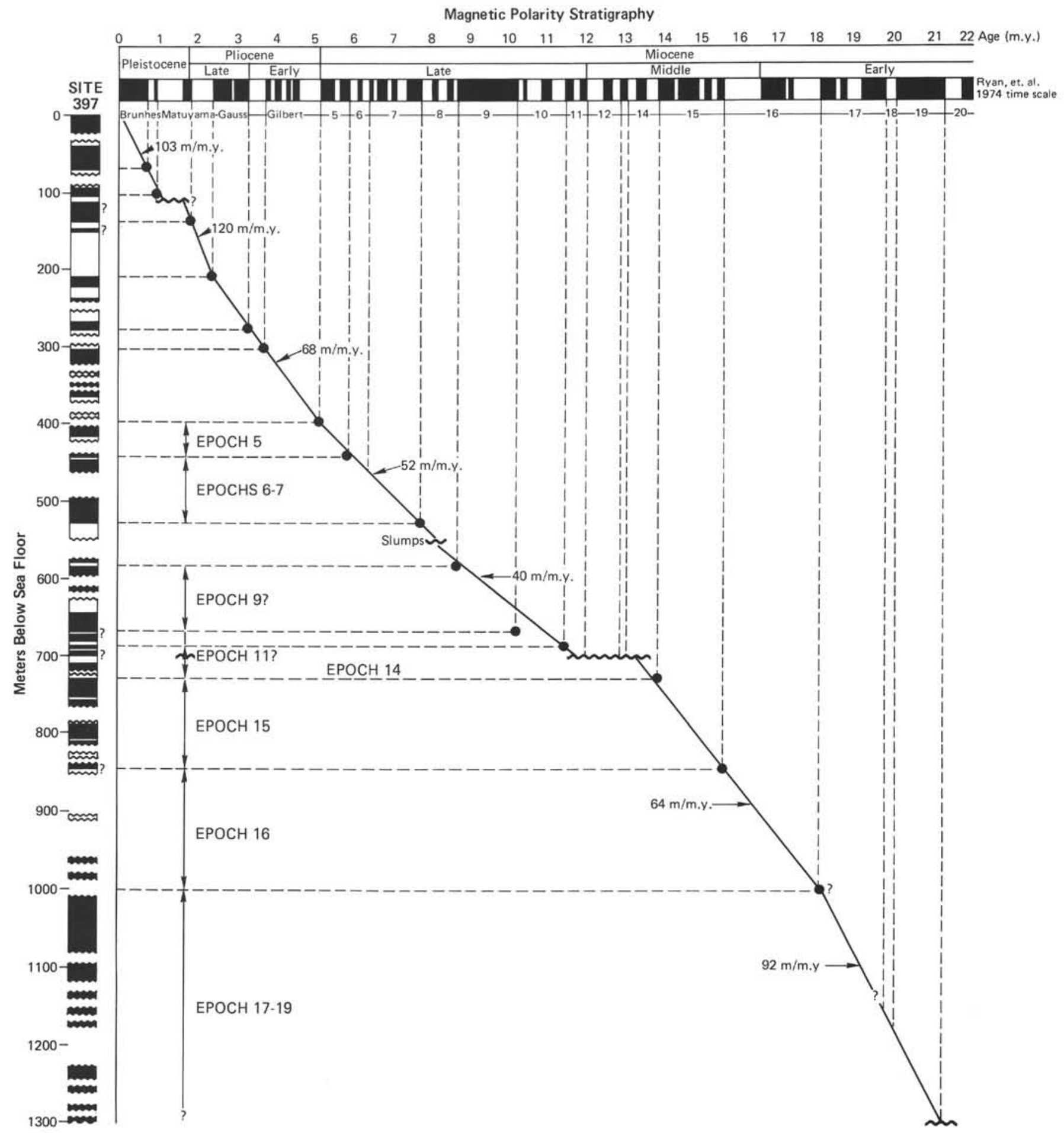

Figure 7. A calibration of Site 397 magnetic polarity reversal sequence to the Ryan et al. (1974) time scale which allows estimates of sediment accumulation rates to be determined.

younger than the postulated reversal detected at Site 397 described above. A few earlier workers (e.g., Ninkovich et al., 1966) cite evidence or give paleomagnetic data from deep-sea cores which can be interpreted to show the possible existence of polarity reversals older than 110,000 years but younger than the Brunhes/Matuyama boundary. However, there is some uncertainty as to whether these are true reversals or localized magnetic excursions. Clearly in the light of the reversal detected here, some reappraisal is necessary, particularly since high resolution deep-tow magnetic profiles are now available for Brunhes age oceanic crust in the inner rift valley floor of the FAMOUS area (MacDonald, 1977) within which reversally polarized 
blocks have been detected. The amplitude changes evident on such marine magnetic profiles which may reflect geomagnetic field intensity fluctuations during the Brunhes Epoch may also be preserved in the paleomagnetic record of rapidly sedimented margins such as that off northwestern Africa.

\section{Early Cretaceous Sediments}

Apart from the thin reversed zone in the upper part of the Early Cretaceous sequence at Site 397, the polarities of remaining sediments are normal (see Figure 5). Calibration to the van Hinte (1976) Cretaceous time scale is not unequivocal. According to the biostratigraphic studies on these sediments, the upper 14 meters of the sequence are dated as early Barremian but absolute biozonation is uncertain. It is at this level that the reversed polarity interval occurs. On the basis of van Hinte's compilation then, this reversed zone could represent either the reversed interval which is identified as marine magnetic anomaly $M 1$ or the shorter $M 2$ anomaly.

It is difficult to reconcile such an assignment of polarity stratigraphy with the biostratigraphic dating of the underlying 151 meters of sediment as late Hauterivian. This was dominantly a time of reversed polarity according to the van Hinte (1976) time scale, whereas the polarity sequence observed here in these sediments is predominantly normal. A more plausible correlation may be found by assigning these normal polarities to the longer normal interval between magnetic anomalies $M 3$ and $M 5$, inferring a correlation of the upper reversed zone with the $M 4$ magnetic anomaly. If correct, this interpretation of the magnetic stratigraphy implies a chronological assignment of the whole sequence to uppermost early Hauterivian instead of late Hauterivian-early Barremian. As these sediments are referred mainly to a single biozone (Cruciellipsis cuvillieri), this biozone can be now calibrated fairly precisely to the absolute time scale between 123 and 124 m.y. B.P. All this evidence points to accumulation of these laminated mudstones under rapid conditions with sedimentation rates in excess of $100 \mathrm{~m} / \mathrm{m}$.y.

\section{Magnetic Susceptibility and NRM Intensity Variations}

The bulk susceptibility of marine sediments reflects variation in magnetic mineral content. At times when influx of terrigenous detritus (particularly that derived from igneous or crystalline basement terrains) is increased, the susceptibility of accumulating sediment increases in magnitude also. In a passive continental margin setting incursion of such detritus is often a significant contribution to the developing sedimentary prism. At other times when terrigenous input is diminished and high biogenic productivity prevails, the sediments will be characterized by low susceptibility. Magnetic intensity changes are caused not only by lithological changes, or change in remanence carriers and type of magnetization, but may also reflect variation in paleofield intensity at the time of remanence acquisition.
At Site 397, the downhole variation in susceptibility and NRM intensity exhibits some interesting correlation with predominant sediment lithologies (Figure 8). The high susceptibilities and intensities of the volcaniclastic sandstones of the middle Miocene emerge as a major feature.

Below the major unconformity, the Early Cretaceous mudstones are associated with fluctuating values of both parameters superimposed on a trend of decreasing value uphole. This could indicate increasing maturity of the distal prodelta slope environment. Above the hiatus, the results of susceptibility and intensity determinations for early Miocene sediments (between 850 and 1298 sub-bottom), reflects a sampling bias towards the marly limestone sub-facies in preference to chaotic pebbly mudstone facies; on the whole, moderately low susceptibilities and intensities are found. The onset of the volcaniclastic sandstone horizons is marked by abrupt increases in susceptibility and intensity which reach maximum values of $1 \times 10^{-3} \mathrm{G} / \mathrm{Oe}$ and $6 \times 10^{-4} \mathrm{G}$, respectively. The trend above this level for the late Miocene sediments is decreasing values of both parameters.

The hemipelagic sediments of Pliocene and early Pleistocene age are marked by some constancy of susceptibility value $\left(4 \times 10^{-6} \mathrm{G} / \mathrm{Oe}\right)$, apart from minor fluctuations. However, there is a displacement to smaller values of susceptibility following the Jaramillo event in the early Pleistocene, before the somewhat high values of the uppermost cores are encountered. Briskin and Berggren (1975) recognize from detailed faunal studies of an equatorial Atlantic core that the late Jaramillo marks the transition between the two major climatic regions of the Pleistocene. The susceptibility results described here appear to confirm the Jaramillo as a significant climatic marker. An increase in magnetic intensity characterizes the sediments of the late Pliocene and early Pleistocene. The comparatively higher intensities of the lowermost Brunhes Epoch show a decrease following the inferred reversed polarity zone. An anomalously high intensity is encountered in the uppermost core. When the intensity variation is viewed overall, there appears to be a long-term cyclical change, associated with a periodicity of approximately 15 million years. Further study is necessary to examine the reality of this.

\section{CONCLUSIONS}

Preliminary paleomagnetic study on Cenozoic and Mesozoic sediments recovered at Site 397 off Cape Bojador have enabled a fairly detailed magnetic polarity stratigraphy to be recognized for part of the northwest African continental margin. The 1300-meter-thick Cenozoic sediment sequence comprises strata of Brunhes to Epoch $17 / 19$ in age. There are three possible hiatuses within this sequence; one of which, in the early Pleistocene, is confirmed on the basis of evidence from the magnetic stratigraphy.

Correlation of the magnetic stratigraphy to an established geomagnetic time scale enables quantitative esti- 

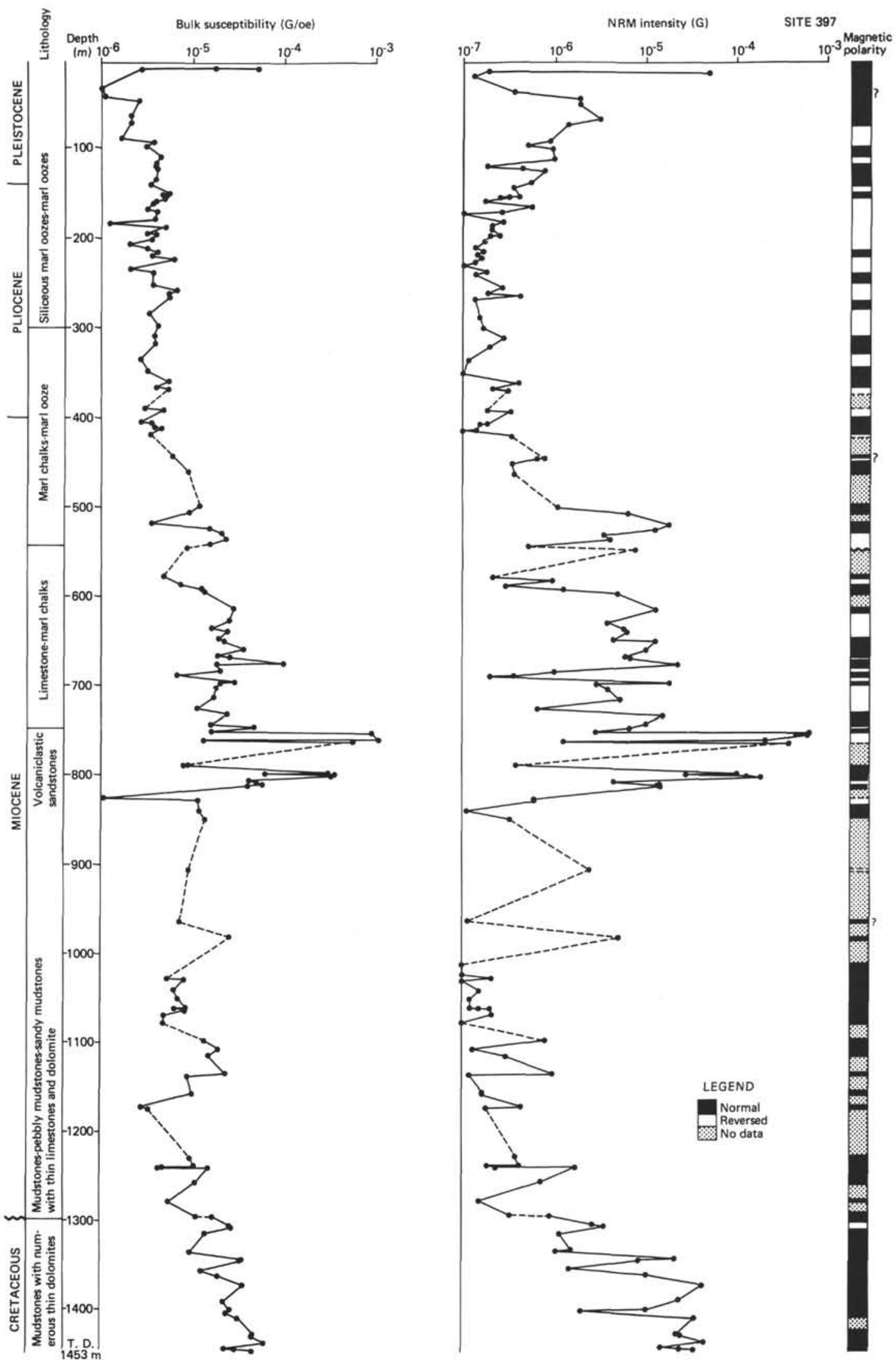

Figure 8. Downhole magnetic susceptibility and NRM intensity variation. 
mates of sediment accumulated rates to be made. These confirm the rapidity of the early Miocene phase of upbuilding of the continental margin and the high rates of accumulation of hemipelagic biogenous sediments during late Neogene and Quaternary times.

Preliminary intepretations of the predominant normal polarities encountered for the Early Cretaceous sediment suggest correlation within the interval between the M3 and M5 Mesozoic marine magnetic anomalies, dated as early Hauterivian.

These studies demonstrate that deciphering of the magnetic stratigraphy in continuously cored sequences can be readily achieved. The magnetic stratigraphy complements biostratigraphic dating and together provide for a more complete understanding of the chronology of the evolution of passive continental margins than from either alone. Nevertheless, it must be emphasized that gaps in sample coverage can give rise to some ambiguity in correlation of the magnetic stratigraphy to established polarity time scales.

\section{ACKNOWLEDGMENTS}

I am grateful to Lindsay Molyneux for providing a newly designed tumbler system and demagnetizing coil for use onboard Glomar Challenger. I thank David Spanner for assistance with shore-based measurements. I acknowledge encouragement and interest from Professor F. Hodson and generous use of departmental facilities. Ernest A. Hailwood reviewed the manuscript and suggested some useful improvements.

\section{REFERENCES}

Ade-Hall, J. M. and Johnson, H. P., 1976. Paleomagnetism of sediments, Leg 34. In Yeats, R. S., Hart, S. R., et al., Initial Reports of the Deep Sea Drilling Project, v. 34: Washington (U.S. Government Printing Office), p. 533-539.

Amerigian, C. and Watkins, N. D., 1975. Chemical overprinting of upper Cenozoic sediments (abstract), IAGA Bull. 36 , p. 164 .

Berggren, W. A., McKenna, M. C., Hardenbrol, J., and Obradovich, J. D., 1978. Revised Paleogene polarity time scale, J. Geol., v. 86, p. 67-81.

Blow, R. A. and Hamilton, N., 1975. Palaeomagnetic evidence from DSDP cores of northward drift in India, $\mathrm{Na}$ ture, v. 257 , p. $570-572$.

Briskin, M. and Berggren, W. A., 1975. Pleistocene stratigraphy and quantitative paleo-oceanography of tropical North Atlantic core V16-205. In Saito, T. (Ed.), Late Neogene Epoch Boundaries: Micropaleontology Special Publ. No. 1, p. 167-198.
Gartner, S. 1977. Calcareous nannofossil biostratigraphy and revised zonation of the Pleistocene, Marine Micropaleontol., v. 2, p. 1-25.

Gartner, S. and Emiliani, C., 1976. Nannofossil biostratigraphy and climatic stages of Pleistocene Brunhes Epoch, Am. Assoc. Petrol. Geol. Bull., v. 60, p. 1562-1564.

Hailwood, E. A. and Hamilton, N., 1978. Quaternary geomagnetic secular variation and polarity reversal record at DSDP Sites 379 and 380, Black Sea. In Ross, D. A., Neprochnov, Y. P., et al., Initial Reports of the Deep Sea Drilling Project, v. 42, Part 2: Washington (U.S. Government Printing Office), p. 1069-1076.

Hamilton, N., 1974. Paleomagnetic measurements Site 222. In Whitmarsh, R. B., Weser, O. E., et al., Initial Reports of the Deep Sea Drilling Project, v. 23: Washington (U.S. Government Printing Office), p. 221-222.

Henry, K. W. and Opdyke, N. D., 1970. Paleomagnetism of specimens from Leg 3 of the Deep Sea Drilling Project. In Maxwell, A. E. et al., Initial Reports of the Deep Sea Drilling Project, v. 3: Washington (U. S. Government Printing Office), p. 667-696.

La Brecque, J. L., Kent, D. V., and Cande, S. C., 1977. Revised magnetic polarity time scale for Late Cretaceous and Cenozoic time, Geology, v. 5, p. 330-335.

Molyneux, L., 1971. A complete results magnetometer for measuring the remanent magnetization of rocks, Geophys. J. Roy Astron. Soc., v. 24, p. 429-433.

MacDonald, K. C., 1977. Near bottom magnetic anomalies, asymmetric spreading, oblique spreading and tectonics of the Mid-Atlantic Ridge near Lat. $37^{\circ} \mathrm{N}$, Geol. Soc. Am. Bull., v. 88 , p. 541-555.

McDougall, I., Saemundsson, K., Johannesson, H., Watkins, N. D., and Kristjansson, L., 1977. Extension of the geomagnetic polarity time scale to 6.5 m.y.: K-Ar dating, geological and paleomagnetic study of a $3,500 \mathrm{~m}$ lava succession in western Iceland, Geol. Soc. Am. Bull., v. 88, p. $1-15$.

Ninkovich, D., Opdyke, N. D., Heezen, B. C., and Foster, J. H., 1966. Paleomagnetic stratigraphy, rates of deposition and tephrachronology in north Pacific deep-sea sediments, Earth Planet. Sci. Lett., v. 1, p. 476-492.

Ryan, W. B. F., Cita, M. B., Dreyfus Rawson, M., Burkle, L. H., and Saito, T., 1974. A paleomagnetic assignment of Neogene stage boundaries and the development of isochronous datum planes between the Mediterranean, the $\mathrm{Pa}$ cific and Indian oceans in order to investigate the response of the world ocean to the Mediterranean salinity crisis, Riv. Ital. Paleontol., v. 80, p. 631-688.

Tarling, D.H. and Mitchell, J.G., 1976. Revised Cenozoic polarity time scale, Geology, v. 4, p. 133-136.

van Hinte, J. E., 1976. A Cretaceous time scale, Am. Assoc. Petrol. Geol. Bull., v. 60, p. 498-516.

Wilson, R. L., 1970. Permanent aspects of the Earth's nondipole magnetic field over Upper Tertiary times, Geophys. J. Roy. Astron. Soc., v. 19, p. 417-438. 